\title{
Silver nanoparticles: aggregation behavior in biorelevant conditions and its impact on biological activity
}

This article was published in the following Dove Medical Press journal: International Journal of Nanomedicine

\author{
Péter Bélteky' \\ Andrea Rónavári ${ }^{1,2}$ \\ Nóra Igaz ${ }^{2}$ \\ Bettina Szerencsés ${ }^{3}$ \\ Ildikó Y Tóth' \\ Ilona Pfeiffer ${ }^{3}$ \\ Mónika Kiricsi² \\ Zoltán Kónya ${ }^{1,4}$
}

'Department of Applied and Environmental Chemistry, Faculty of Science and Informatics, University of Szeged, Szeged, Hungary; ${ }^{2}$ Department of Biochemistry and Molecular Biology, Faculty of Science and Informatics, University of Szeged, Szeged, Hungary; ${ }^{3}$ Department of Microbiology, Faculty of Science and Informatics, University of Szeged, Szeged, Hungary; ${ }^{4}$ MTA-SZTE Reaction Kinetics and Surface Chemistry Research Group, Szeged, Hungary
Correspondence: Zoltán Kónya Department of Applied and Environmental Chemistry, Faculty of Science and Informatics, University of Szeged, Rerrich Square I, H-6720 Szeged, Hungary

Tel +36 62544620

Email konya@chem.u-szeged.hu
Purpose: The biomedical applications of silver nanoparticles (AgNPs) are heavily investigated due to their cytotoxic and antimicrobial properties. However, the scientific literature is lacking in data on the aggregation behavior of nanoparticles, especially regarding its impact on biological activity. Therefore, to assess the potential of AgNPs in therapeutic applications, two different AgNP samples were compared under biorelevant conditions.

Methods: Citrate-capped nanosilver was produced by classical chemical reduction and stabilization with sodium citrate $(\mathrm{AgNP} @ \mathrm{C})$, while green tea extract was used to produce silver nanoparticles in a green synthesis approach (AgNP@GTs). Particle size, morphology, and crystallinity were characterized using transmission electron microscopy. To observe the effects of the most important biorelevant conditions on AgNP colloidal stability, aggregation grade measurements were carried out using UV-Vis spectroscopy and dynamic light scatterig, while MTT assay and a microdilution method were performed to evaluate the effects of aggregation on cytotoxicity and antimicrobial activity in a time-dependent manner.

Results: The aggregation behavior of AgNPs is mostly affected by $\mathrm{pH}$ and electrolyte concentration, while the presence of biomolecules can improve particle stability due to the biomolecular corona effect. We demonstrated that high aggregation grade in both AgNP samples attenuated their toxic effect toward living cells. However, AgNP@GT proved less prone to aggregation thus retained a degree of its toxicity.

Conclusion: To our knowledge, this is the first systematic examination regarding AgNP aggregation behavior with simultaneous measurements of its effect on biological activity. We showed that nanoparticle behavior in complex systems can be estimated by simple compounds like sodium chloride and glutamine. Electrostatic stabilization might not be suitable for biomedical AgNP applications, while green synthesis approaches could offer new frontiers to preserve nanoparticle toxicity by enhancing colloidal stability. The importance of properly selected synthesis methods must be emphasized as they profoundly influence colloidal stability, and therefore biological activity.

Keywords: colloidal stability, green synthesis, antimicrobial activity, cytotoxicity

\section{Introduction}

Due to the numerous favorable properties of nanomaterials, their biomedical application is expanding drastically. ${ }^{1,2}$ Silver nanoparticles (AgNPs) exhibit excellent antimicrobial and cytotoxic activities, rendering them one of the most frequently utilized nanomaterials. ${ }^{3-6}$ The physical, chemical, and biological features of AgNPs are determined by a number of factors like nanoparticle size and morphology or by the nature of the capping materials covering the particle surface. ${ }^{7,8}$ Therefore, proper selection 
of synthesis methods - most importantly via adequate reducing and stabilizing agents - is crucial to achieve the desired particle properties. ${ }^{9,10}$

Recently, a number of synthesis methods for AgNP production have been developed, nevertheless, the most common approach is the chemical reduction of a silver salt by organic or inorganic compounds using various capping agents to prevent agglomeration. ${ }^{11}$ Although chemical reduction methods are easy to control and are effective in achieving high monodispersity and shape specificity, they are relatively expensive and often involve toxic chemicals that have unforeseen consequences toward the environment and human health. ${ }^{12,13}$ As cost-effective and eco-friendly alternatives, biological and green synthesis methods have become the center of attention in recent years. While these approaches offer less direct control over size and morphology, they utilize mild experimental conditions and carefully selected, nontoxic, environmentally benign solvents, reducing and capping materials. Green strategies frequently employ either living organisms or natural extracts since these contain a plethora of biodegradable, generally nontoxic biomolecules that can serve both as reducing and stabilizing agents. ${ }^{12,14,15}$ Plant extracts offer a large variety of antioxidants that could contribute to nanoparticle formation in various ways. ${ }^{16}$ For instance, flavonoid-rich plant extracts proved to be excellent candidates for silver ion reduction, according to a variety of different publications. ${ }^{17-19}$ Furthermore, phenolic acid-type caffeine and theophylline found in hydroalcoholic extracts of Coffea arabica, Camellia sinensis and black tea leaf extracts have been utilized in previous research as stabilizing agents. ${ }^{20,21}$ About $30 \%-40 \%$ of solid components in green tea is (-)-epigallocatechin gallate, a compound which can act both as a reducing and as a stabilizing agent for nanoparticles due to its unique properties. ${ }^{16,22}$

Although numerous studies have been published on nanoparticle synthesis methods, none of them examined systematically the aggregation behavior of the generated particles while simultaneously addressing the impact of nanoparticle aggregation on biological activity. In fact, the colloidal stability of nanoparticles in living systems (in part defined by the applied synthesis method) can directly influence biological activity, which issue cannot be overlooked, especially when these materials are designed for therapeutic utilizations. Elzey and Grassian presented results regarding the agglomeration of bare AgNPs under environmentally relevant conditions. ${ }^{23}$ Although the use of AgNPs without any surface coating and the absence of salts in the liquid phase are ideal circumstances to examine particle aggregation, the addition of further components and the analysis of their effects on this nanoparticle feature could be utilized to approximate the behavior of the particles within living systems more adequately. El Badawy et al investigated the effect of environmental conditions like $\mathrm{pH}$, ionic strength, and electrolyte type on the aggregation behavior of different AgNP suspensions with the help of dynamic light scattering (DLS) measurements. ${ }^{24,25}$ They concluded that electrosterically stabilized AgNPs are less prone to aggregate under experimental conditions compared to uncoated and electrostatically stabilized systems, ${ }^{24,25}$ however, biomolecule interactions and the effect of nanoparticle aggregation on toxicity were not the scope of their research. It is well known that nanomaterials can interact with various biomolecules of living organisms, most prominently with proteins, that are able to adsorb on nanoparticle surfaces, establishing the so-called biomolecular corona. ${ }^{26,27}$ This corona serves as a kind of biological identity of the nanoparticle; moreover, it contributes to colloidal stability as the protein-rich corona can counteract the abovementioned disruptive environmental conditions, also demonstrated by Tai et al and Alarcon et al. ${ }^{28,29}$ In our recently published papers, we described environmentally benign synthesis approaches for green nanomaterial formation, and we also showed that the various green materials used for stabilization and for reduction of metal ions have a defining role in determining and fine-tuning the biological activity of the obtained green nanoparticles. ${ }^{15,30,31}$

The scientific literature is lacking in data on the aggregation behavior, and especially its relation to the biological properties of AgNPs, as a systematic examination involving a wide variety of parameters has never been performed. Therefore, in this paper we executed a thorough analysis of this issue. Two different AgNP samples with comparable sizes and morphologies were synthesized. One was produced with the most common classical chemical method: a citrate-capped nanosilver (AgNP@C) reduced by sodium borohydride, the other (green tea extract-stabilized silver nanoparticles [AgNP@GTs]) was obtained with an eco-friendly and lowcost, green approach using green tea extract as reducing and stabilizing agent. Our aim was to examine methodically, under biorelevant conditions, the effects of $\mathrm{pH}$ and differing concentrations of sodium chloride, glucose, glutamine, and FBS - both in water and in DMEM - on the aggregation behavior of these particles in a time-dependent manner in order to evaluate the nanoparticle aggregation grade and its impact on cytotoxicity.

\section{Materials and methods AgNP@Cs}

AgNP@Cs were synthesized by chemical reduction using sodium borohydride $\left(\mathrm{NaBH}_{4}\right.$; EMD Millipore, Billerica, MA, 
USA) as reported by Wan et al, with slight modifications. ${ }^{32}$ First, $0.2 \mathrm{~g}$ of sodium citrate $\left(\mathrm{Na}_{3} \mathrm{C}_{6} \mathrm{H}_{5} \mathrm{O}_{7} \times 2 \mathrm{H}_{2} \mathrm{O}\right.$; EMD Millipore) was dissolved in $75 \mathrm{~mL}$ deionized water with vigorous stirring. The solution was heated to $70^{\circ} \mathrm{C}$ and $2 \mathrm{~mL}$ of $1 \mathrm{w} / \mathrm{v} \%$ silver nitrate $\left(\mathrm{AgNO}_{3}\right.$; EMD Millipore), then $2 \mathrm{~mL}$ of $0.1 \mathrm{w} / \mathrm{v} \% \mathrm{NaBH}_{4}$ solution were added dropwise into the mixture. The resulting yellowish brown suspension was stirred at $70^{\circ} \mathrm{C}$ for 1 hour.

Removal of any interfering borohydride residues was carried out by dialysis against distilled water for 24 hours using cellulose membrane. After the purification step, $0.02 \mathrm{~g}$ sodium citrate was added into the mixture to compensate any capping agent removed during dialysis.

\section{AgNP@GTs}

AgNP@GTs were synthesized by the method previously reported by Rónavári et al with slight adjustments. ${ }^{30}$ Briefly, $2 \mathrm{~g}$ of dry green tea leaves (R. Twining and Company Limited, London, England) were soaked in $100 \mathrm{~mL}$ deionized water to prepare a primary extract. The dispersion was heated at $80^{\circ} \mathrm{C}$ for 20 minutes. Afterwards the extract was cooled, vacuum-filtered using nylon membrane of $0.45 \mu \mathrm{m}$ pore diameter (Maine Manufacturing LLC, Sanford, ME, USA), and this filtrate was further used both as a reducing and as a stabilizing agent for AgNPs.

AgNPs were synthesized by mixing the green tea extract with $0.1 \mathrm{M}$ aqueous silver nitrate solution in 2:1 volume ratio at room temperature by constant stirring for 24 hours and later filtered using a polyvinylidene fluoride syringe filter with $0.22 \mu \mathrm{m}$ pore size. The resulting brown suspension was kept at $4^{\circ} \mathrm{C}$ until further use.

\section{Characterization of nanoparticles}

Morphological characteristics like size, aspect ratio, and shape distributions of the obtained samples were examined. After drop casting the samples on carbon film-coated copper grids of 200 mesh grid density, transmission electron microscopic (TEM) images (at least 15 representative images, $\sim 750$ particles) and electron diffraction (ED) patterns were taken with a FEI Tecnai $\mathrm{G}^{2} 20 \mathrm{X}$ Twin instrument (FEI Corporate Headquarters, Hillsboro, OR, USA) using $200 \mathrm{kV}$ accelerating voltage. Particles were measured using ImageJ software and the resulting histograms were constructed by the OriginPro 8.5 (OriginLab, Northampton, MA, USA) software package. . $^{334}$

To interpret the initial colloidal stability of the samples prior to the aggregation measurements, the average hydrodynamic diameters (Z-average), zeta potentials ( $\zeta$-potential), and lastly their characteristic surface plasmon resonance
(SPR) were measured. The average hydrodynamic diameter of the samples was assessed at $37^{\circ} \mathrm{C} \pm 0.1^{\circ} \mathrm{C}$ using DLS analysis on a Malvern Zetasizer Nano ZS instrument (Malvern Instruments, Malvern, UK) using disposable capillary cells. To measure zeta potential, we used the second- or third-order cumulant fit of the autocorrelation functions, depending on the degree of polydispersity. The electrophoretic mobility of the samples was measured at $37^{\circ} \mathrm{C} \pm 0.1^{\circ} \mathrm{C}$, subsequently the Smoluchowski equation was applied to convert electrophoretic mobility to zeta potential values, with the accuracy of $\pm 5 \mathrm{mV}$. Characteristic SPR properties were obtained by $\mathrm{UV}-\mathrm{Vis}$ spectroscopy. The absorbance spectra of the asprepared nanoparticles were recorded within the range of 250-800 nm with distilled water as background for AgNP@C and appropriately diluted green tea for AgNP@GT using an Ocean Optics 355 DH-2000-BAL UV-Vis spectrophotometer (Halma PLC, Largo, FL, USA) and a $10 \mathrm{~nm}$ path length quartz cuvette.

To determine the exact interactions between the organic capping agents and nanoparticles, Raman spectroscopic measurements were performed using a Senterra II Raman microscope (Bruker, Billerica, MA, USA) at $532 \mathrm{~nm}$ excitation wavelength, $12.5 \mathrm{~mW}$ laser power, and $4 \mathrm{~cm}^{-1}$ spectral resolution.

\section{Aggregation behavior experiments}

To investigate the aggregation behavior of the synthesized AgNP samples, the constant AgNP concentration of $80 \mathrm{ppm}$ was selected and consistently applied throughout the experiments, and the most important biorelevant agents and conditions were considered. The effect of different $\mathrm{pH}$ values, different sodium chloride, glucose, and glutamine concentrations, moreover - as model systems for biological fluids - the effect of DMEM and FBS (both in water and in DMEM) was investigated as shown in Table 1. Time dependence of the aggregation was also examined; measurements were carried out at the $0,1.5,3,6,12$, and 24 hours checkpoints.

Particle aggregation was tested at different sodium chloride $(10,50$, and $150 \mathrm{mM})$ and glucose $(3.9$ and $6.7 \mathrm{mM})$ concentrations and at various $\mathrm{pH}$ values (set to $3,5,7.2$, or 9). These values

Table I List of the investigated biorelevant conditions

\begin{tabular}{l|l}
\hline Condition & Values \\
\hline $\mathrm{pH}$ & $3,5,7.2^{*}, 9$ \\
$\mathrm{NaCl}$ concentration (mM) & $10,50,150$ \\
Glucose concentration (mM) & $3.9,6.7$ \\
Glutamine concentration (mM) & 4 \\
DMEM (v/v\%) & 45 \\
FBS (v/v\%, both in water and in DMEM mixture) & 5 \\
\hline
\end{tabular}

Notes: Reference point for the measurements is marked with $(*)$. All samples were measured under $37^{\circ} \mathrm{C}$; furthermore, $\mathrm{pH} \sim 7.2$ and $10 \mathrm{mM} \mathrm{NaCl}$ were utilized as background, unless otherwise stated. 
were selected according to similar characteristic values observed by mammalian species. ${ }^{35-37}$ The glutamine concentration was set to $4 \mathrm{mM}$ as it is the most commonly utilized concentration in cell culture experiments. ${ }^{38}$ Our preliminary viability assays indicated that the medium (consisting originally of DMEM with $10 \mathrm{v} / \mathrm{v} \% \mathrm{FBS}$ ) can be diluted only moderately (maximum 1:1 with aqueous solutions of interfering agents such as various inorganic salts) without risking osmotic damage of the cells.

All of our samples - with the exceptions of certain $\mathrm{pH}-$ and $\mathrm{NaCl}$-related measurements (seen in the first two rows of Table 1) - were examined with a constant background salt concentration of $10 \mathrm{mM} \mathrm{NaCl}$ at $\mathrm{pH} 7.2$ and at $37^{\circ} \mathrm{C}$. Experiments involving DMEM were not adjusted due to its already complex composition. The sample corresponding to the pH 7.2 measurement, marked with * in Table 1, was used as reference point.

A typical measurement would pursue the following procedure: first, the samples were prepared in $5 \mathrm{~mL}$ volumes with the conditions set according to Table 1. Initially, the AgNP samples and the biorelevant agents were mixed and diluted to $4 \mathrm{~mL}$ and the remaining volume was utilized to set the appropriate $\mathrm{pH}$. Afterwards, the mixtures were sonicated on $37 \mathrm{kHz}$ for 30 seconds which marked the starting point of the experiments. DLS data regarding particle size and UV-Vis data were gathered simultaneously while the zeta potential values were obtained right after the size measurements. Between each measurement the samples were kept at $37^{\circ} \mathrm{C}$, and prior to the actual measurement, they were homogenized by manual shaking and their $\mathrm{pH}$ values were readjusted if necessary.

\section{Cytotoxicity assays}

Human lung adenocarcinoma (A549) and human lung noncancerous fibroblast (MRC-5) cell lines were purchased from ATCC and were maintained in DMEM containing $1 \mathrm{~g} / \mathrm{L}$ glucose, supplemented with 10\% FBS, 2 mM L-glutamine, $0.01 \%$ streptomycin, and $0.005 \%$ ampicillin. Cells were cultured under standard conditions in a $37^{\circ} \mathrm{C}$ incubator at $5 \% \mathrm{CO}_{2}$ in $95 \%$ humidity.

To measure the changes in AgNP cytotoxicity affected by particle aggregation in a time-dependent manner, first, $\mathrm{IC}_{50}$ values of the as-prepared silver samples were determined for both the cancerous and the healthy human cell lines. Thereafter, nanosilver aggregation states were formulated by incubating the particles with $150 \mathrm{mM} \mathrm{NaCl}$ for different time spans $(0,1.5,3,6,12$, and 24 hours $)$ prior to cell treatments. Cells were seeded in 96-well plates using $10^{4}$ cells/well density, then on the following day were treated with the appropriate aggregated AgNP samples for 24 hours. After treatments, cells were washed with PBS and incubated with culture medium containing $0.5 \mathrm{mg} / \mathrm{mL}$ MTT reagent (SigmaAldrich Co., St Louis, MO, USA) for 1 hour at $37^{\circ} \mathrm{C}$. The resulting formazan crystals were dissolved in DMSO and absorbance of the samples was measured at $570 \mathrm{~nm}$ using a SPECTROstar Nano plate reader (BioTech-Hungary, Budapest, Hungary). Absorption values of the untreated control samples were considered as 100\% viability. The experiments were carried out three times using four replicates.

\section{Screening of antibacterial and antifungal activity}

First, a microdilution method was used to determine the minimum inhibitory concentration (MIC) of the different nanosilver samples against Cryptococcus neoformans IFM 5844 (IFM; Research Center for Pathogenic Fungi and Microbial Toxicoses, Chiba University), Bacillus megaterium SZMC 6031, and Escherichia coli SZMC 0582 (SZMC: Szeged Microbiology Collection) strains in RPMI 1640 medium (Sigma-Aldrich Co.) using $10^{5}$ cells/mL, respectively. Two fold dilution series of both nanoparticles were made in the 0-75 ppm concentration range. From each cell suspension $50 \mu \mathrm{L}$ aliquots were loaded into the wells of a 96-well microplate and $50 \mu \mathrm{L}$ of individual AgNP dilutions were added to the cells. Suspensions supplemented with $50 \mu \mathrm{L}$ RPMI 1640 were used as growth control. Then microplates were incubated at $30^{\circ} \mathrm{C}$ for 48 hours and the light absorbance of the samples was measured at $620 \mathrm{~nm}$ using SPECTROstar Nano plate reader (BMG LabTech). The experiment was carried out three times always in triplicates.

The antimicrobial activity of the aggregated nanoparticles was tested on the above mentioned strains with the same procedure described in the "Aggregation behavior experiments" section. Briefly, suspensions of $10^{5}$ cells $/ \mathrm{mL}$ were prepared in RPMI 1640 medium. Fifty microliter aliquots from Cr. neoformans, B. megaterium, and E. coli cell suspensions were loaded into the wells of a 96-well microplate and this time $50 \mu \mathrm{L}$ of the individual aggregated nanoparticle samples were added to the cells. Suspensions supplemented with $50 \mu \mathrm{L}$ RPMI 1640 were used as growth control, while suspensions with non-aggregated nanoparticles were used as controls of toxicity. The light absorbance of the samples was measured at $620 \mathrm{~nm}$ using SPECTROstar Nano plate reader (BMG LabTech). The experiments were carried out three times in four replicates.

\section{Results \\ AgNP characterization}

The morphology and particle size distribution of our AgNP samples were determined based on TEM images (Figure 1). It is well known that the biological activity of AgNPs is 
A

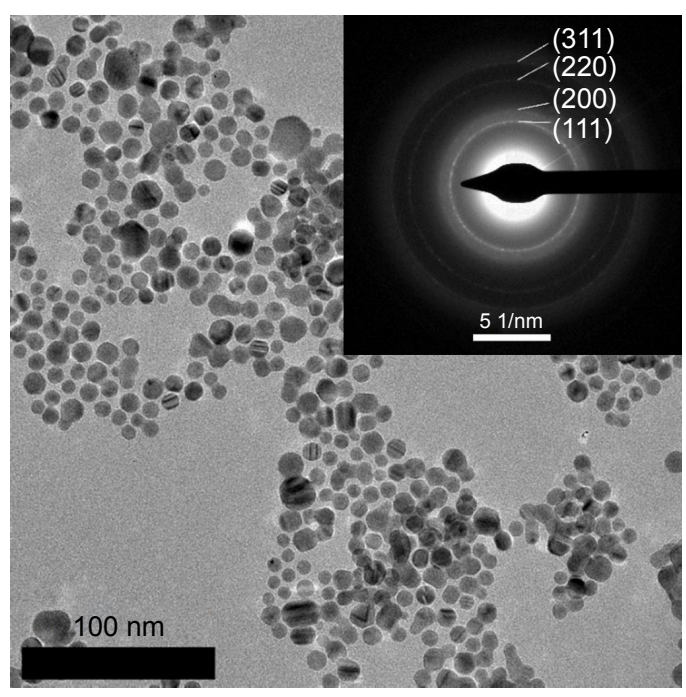

\section{AgNP@C}
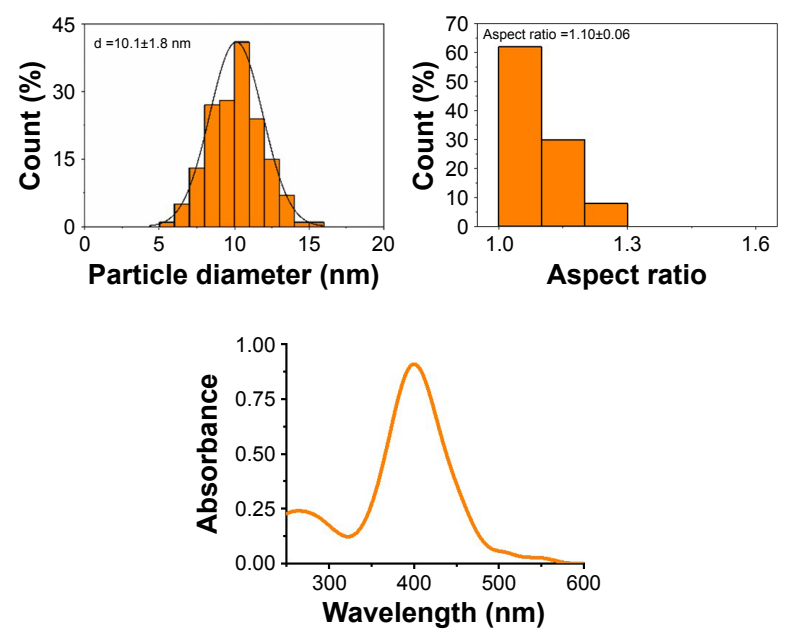

\begin{tabular}{|c|c|c|c|c|c|}
\hline \multirow{2}{*}{ Z-average (d.nm) } & \multirow{2}{*}{ Zeta potential $(\mathrm{mV})$} & \multirow{2}{*}{$\lambda_{\max }(\mathrm{nm})$} & \multicolumn{3}{|c|}{ Shape distribution (\%) } \\
\cline { 4 - 6 } & & & Sphere & Polyhedron & Prism \\
\hline 27.8 & $-27.7 \pm 5$ & 400 & 96.3 & 3.2 & 0.5 \\
\hline
\end{tabular}

B

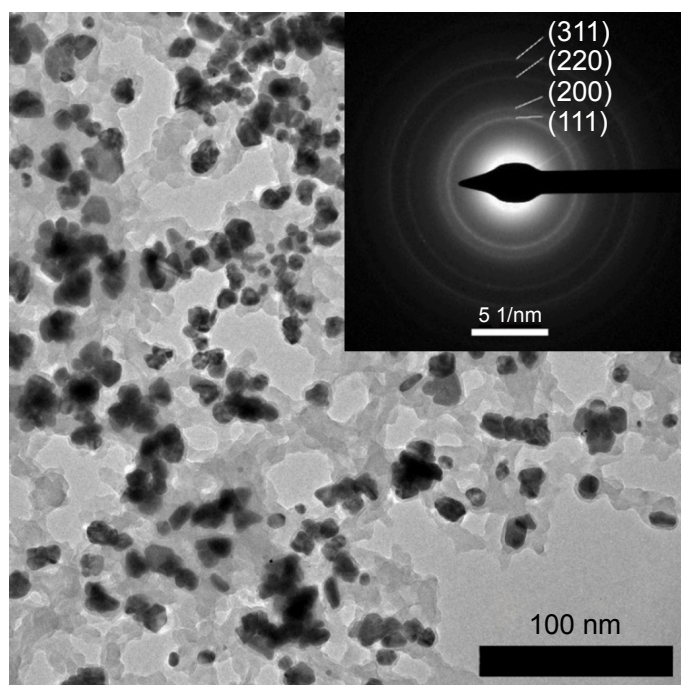

AgNP@GT
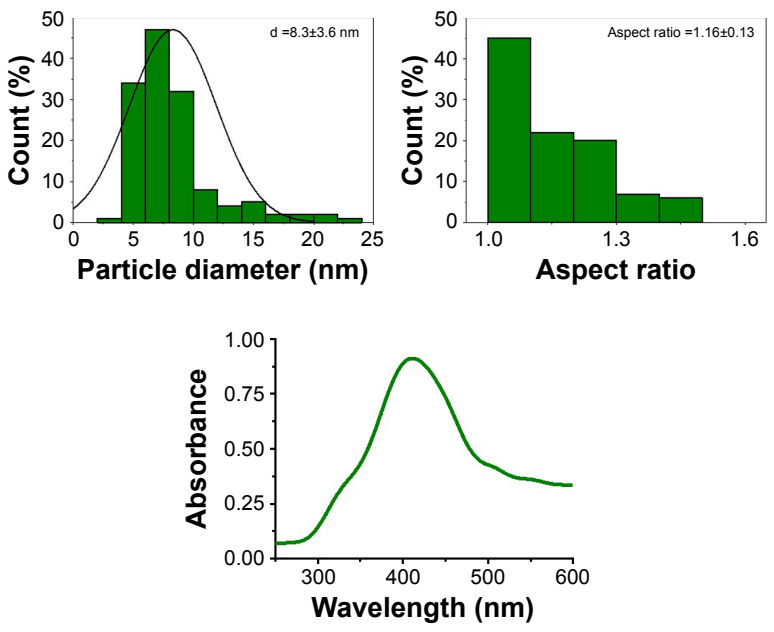

\begin{tabular}{|c|c|c|c|c|c|}
\hline \multirow{2}{*}{ Z-average (d.nm) } & \multirow{2}{*}{ Zeta potential $(\mathrm{mV})$} & \multirow{2}{*}{$\lambda_{\max }(\mathrm{nm})$} & \multicolumn{3}{|c|}{ Shape distribution $(\%)$} \\
\cline { 4 - 6 } & & & Sphere & Polyhedron & Prism \\
\hline 87.7 & $-36.2 \pm 5$ & 411 & 70.1 & 25.2 & 4.7 \\
\hline
\end{tabular}

Figure I TEM images with corresponding size, aspect ratio and shape distributions, electron diffraction patterns, Characterization of silver nanoparticles stabilized (A) by citrate (AgNP@C) and (B) by green tea extract (AgNP@GT) consisting of TEM images with corresponding size, aspect ratio and shape distributions, electron diffraction patterns, UV-Vis spectra with characteristic surface plasmon resonance peaks, furthermore mean values for the average hydrodynamic diameter and zeta potential of the particles ( $\mathrm{pH} \sim 7.2)$.

Abbreviations: AgNP@C, citrate-capped nanosilver; AgNP@GT, green tea extract-stabilized silver nanoparticle; TEM, transmission electron microscopy.

size- and shape-dependent, therefore, our objective was to prepare both nanosilver samples in spherical shape and in a similar size range $(\sim 10 \mathrm{~nm})$ in order to realize comparative chemical and biological analyses. ${ }^{7,39}$
According to TEM images, the classical chemical reduction method (Figure 1A), as well as the environmentally benign green synthesis method (Figure 1B) resulted in similar average sized (AgNP@C $\sim 10.1 \pm 1.8 \mathrm{~nm}$ and 
AgNP@GT 8.3 $\pm 3.6 \mathrm{~nm}$ ) particles, with both methods yielding quasi-spherical nanoparticles, with aspect ratios around 1.10 and 1.16 , respectively. Regarding their exact morphologies, the chemical reduction method was characterized by better shape-specificity compared to the green synthesis approach, as over $96 \%$ of the particles obtained by the classical method proved to be spherical while the green method provided $\sim 70 \%$ spherical particles and a considerable amount of polyhedric- and a low amount of prism-like particles were also present $(25.2 \%$ and $4.7 \%$ respectively, Figure 1B). Nanoparticle shape is important, as different morphologies can arise due to different crystal facets that can affect reactivity and biological activities as well. ${ }^{40,41} \mathrm{ED}$ measurements were carried out to identify possible discrepancies within the crystal lattices of the nanoparticles. The ED results verified the chemical composition and crystallinity of AgNPs by displaying well defined diffraction rings around $4.22,4.96,6.97$, and $8.131 / \mathrm{nm}$ radii that are corresponding to miller indices of $(111,200,220)$ and $(311)$ respectively. ${ }^{30}$ Despite the slight differences in shape specificity, the size, aspect ratio, and crystallinity of the samples showed strong correlation; therefore, the as-prepared AgNPs were deemed comparable for aggregation and biological analysis. The TEM image of AgNP@GT shown in Figure 1B shows nanoparticles in immediate contact with the components of the green tea extract which are essentially embedded in a matrix of various macromolecules present in the biological extract. The presence of these biological matrices in green synthesis methods has been mentioned in a number of previous studies. ${ }^{30,42,43}$

The maximum of the characteristic absorbance spectra of the initial nanosilver suspensions were at $400 \mathrm{~nm}$ for AgNP@C, and at 411 nm for AgNP@GT, respectively (Figure 1). These values are in good agreement with the literature, where spherical AgNPs around $10 \mathrm{~nm}$ have their characteristic absorption peaks around $400 \mathrm{~nm} .^{7}$ The wider SPR peak of the green tea-capped sample underlines the lower particle shape specificity and monodispersity, which is a direct consequence of the more complex, and therefore less controllable nature of the green synthesis method. Even though smaller AgNPs of the same shape tend to have SPR peak maximum values at lower wavelengths, the spectrum of AgNP@GT showed a slightly higher characteristic absorption maximum. ${ }^{7}$ This can also be explained by the presence of a biological matrix surrounding AgNP@GT, as surface interactions between AgNPs and biomolecules can affect their light absorption properties. ${ }^{44}$

DLS measurements were carried out to determine the average hydrodynamic diameter and the zeta potential of the as-prepared samples on $\mathrm{pH} \sim 7.2$. These were used as reference values in aggregation-related measurements. The Z-average value of AgNP@C was 27.8 nm (Figure 1A), whereas that of the particles synthesized by the green method resulted to be $87.7 \mathrm{~nm}$ (Figure 1B). Only the Z-average value of AgNP@C was close to the primary particle size $(10.1 \mathrm{~nm})$. The rather large difference between Z-average and primary size $(8.3 \mathrm{~nm})$ in case of AgNP@GT was most likely due to the expansive nature of the organic matrix surrounding the nanoparticles, which formed a larger hydration layer that resulted in larger average hydrodynamic diameter. On the other hand, the $\zeta$-potential of AgNP@GT was about $8.5 \mathrm{mV}$ lower than the value of the citrate capped particles $(-27.8 \mathrm{mV})$; therefore, a greater stability was expected in the case of AgNP@GT. For both the samples, it can be stated that the negative $\zeta$-potential values are the results of the adsorbed stabilizing agents on the nanoparticle surfaces. These agents have functional groups capable of deprotonation under adequate conditions inducing the observed surface charges. This can be easily realized in the case of AgNP@C where the $-\mathrm{COOH} /-\mathrm{COO}^{-}$equilibrium of the stabilizing citrate is taken into consideration. Furthermore, even though the chemical composition of the green tea extract in AgNP@ GT is much more complex, we can assume largely similar interactions as in the case of AgNP@C, so the carboxyl/ carboxylate model can be used as estimation. ${ }^{45}$

Further Raman spectroscopic characterization was performed to investigate the exact interactions between capping components and AgNPs. Avnir et al offered a methodology for molecularly doped metallic materials. ${ }^{46,47} \mathrm{We}$ found that even in the case of AgNP@GT, where a similar mechanism could occur, the biological compounds of green tea extract are rather adsorbed in a 2D arrangement on the surface of the particles instead of forming molecularly doped materials (Figure S1).

\section{Aggregation behavior Effect of $\mathrm{pH}$}

The effects of different $\mathrm{pH}$ conditions on aggregation behavior of the two AgNP samples prepared by different synthesis methods are shown in Figures 2 and S2. In line with previous studies, both AgNP@C and AgNP@GT samples were more stable on alkaline and neutral than on acidic $\mathrm{pH}$, indicated by smaller Z-average values, more negative zeta potentials and smaller changes in the SPR peaks. ${ }^{24,25}$ With increasing $\mathrm{pH}$, the free organic functional groups on the NP surfaces reach a higher degree of deprotonation, thus the amount of negative surface charge increases simultaneously. This would enhance the electrostatic repulsion between particles 
A

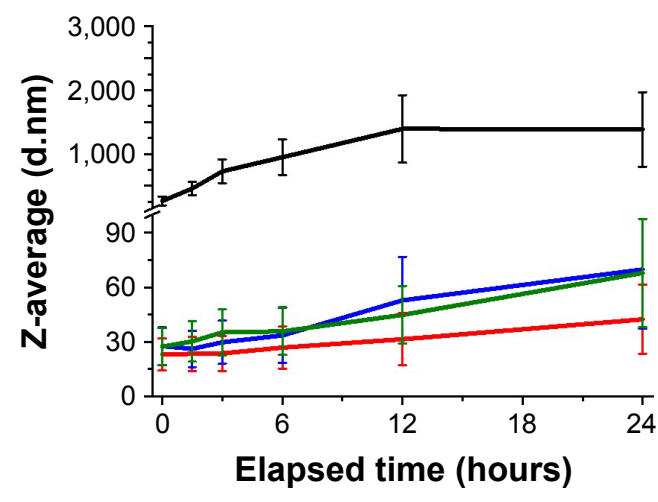

$\mathrm{pH}=3$

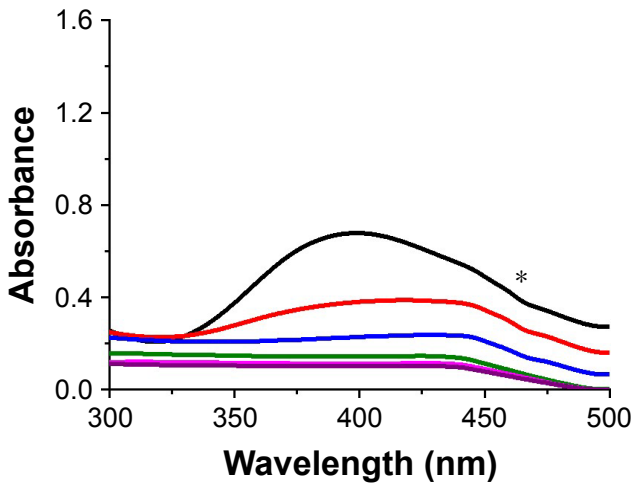

AgNP@C
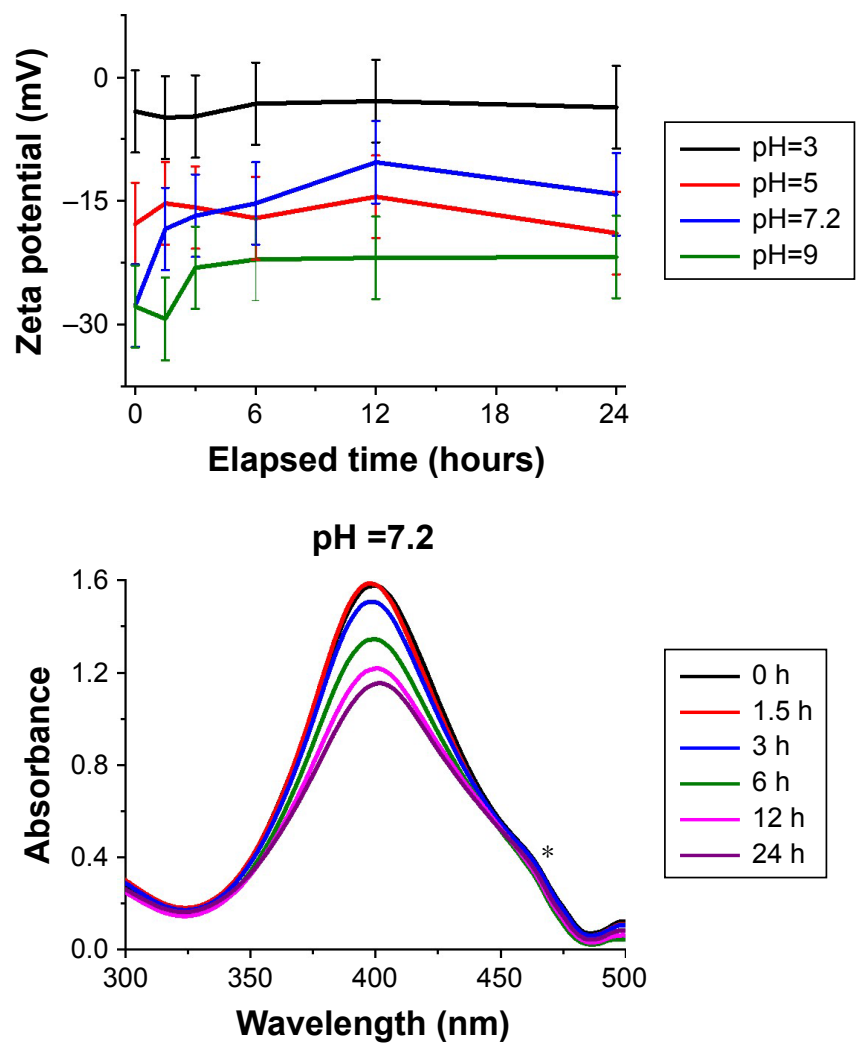

B

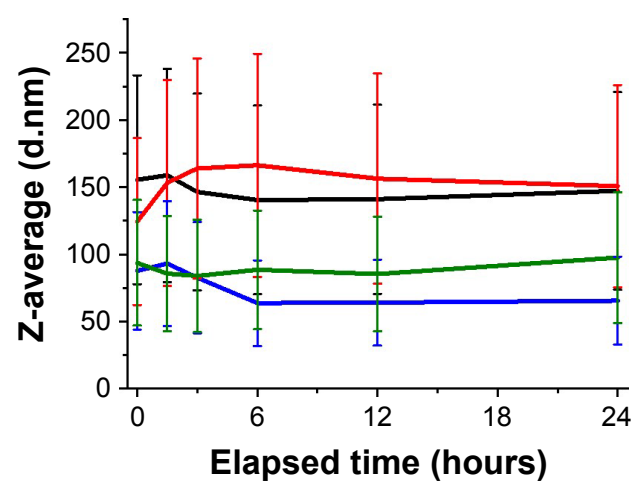

$\mathrm{pH}=3$

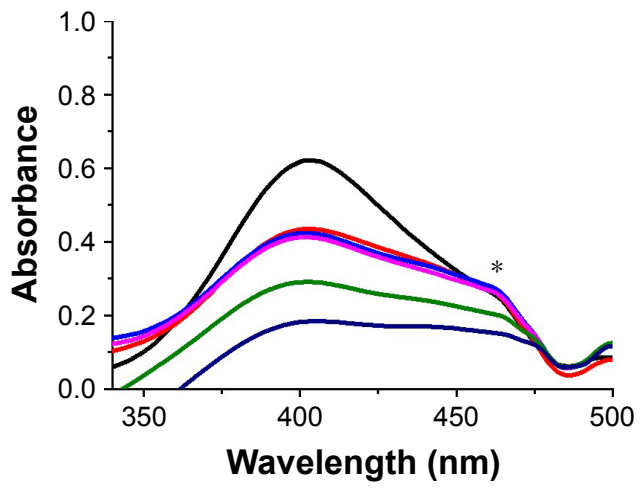

AgNP@GT
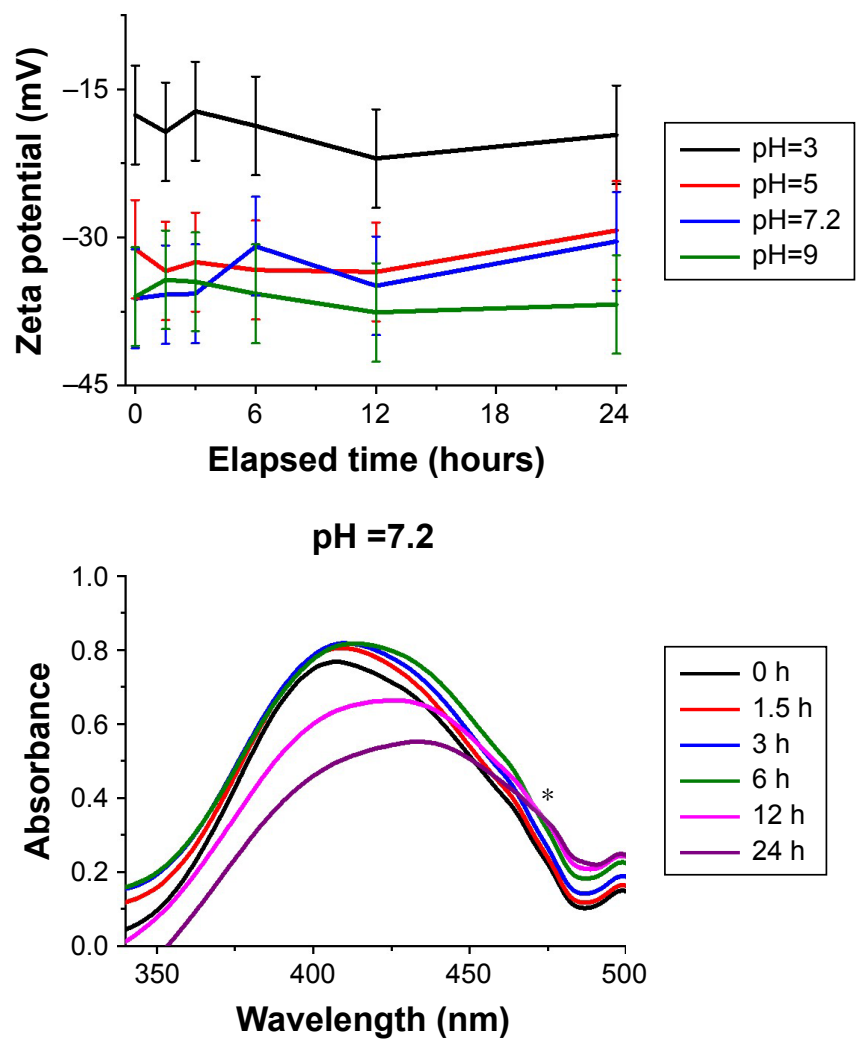

Figure 2 The effect of $\mathrm{pH}$ on the aggregation behavior of the as-prepared silver nanoparticles with $10 \mathrm{mM} \mathrm{NaCl}$ background concentration.

Notes: Average hydrodynamic diameter (Z-average) trend, zeta potential, and UV-Vis spectrum changes of $(\mathbf{A})$ citrate-stabilized AgNP@C, (B) green tea-stabilized AgNP@ GT, observed over 24 hours. * marks a UV-Vis detection error during the measurements that should be disregarded. Abbreviations: AgNP@C, citrate-capped nanosilver; AgNP@GT, green tea extract-stabilized silver nanoparticle. 
resulting in smaller aggregate diameters, explaining the lower Z-average values compared to the measurements on acidic conditions.

AgNP@C (Figure 2A) showed a small gradual increase in hydrodynamic diameter even on alkaline $\mathrm{pH}$, indicating that the background electrolyte concentration of $10 \mathrm{mM} \mathrm{NaCl}$ was enough to initiate slow aggregation in the sample. In comparison, in the case of AgNP@GT no time-dependence of aggregation could be observed (Figure 2B), implying that after the initial experimental criteria have been set, the biological matrix prohibited ongoing changes within the sample, as the system reached an equilibrium state. Interestingly, the green tea extract was capable of limiting the excessive growth of the aggregates, since even the biggest aggregates in the AgNP@GT sample were below 250 nm, while Z-average values of AgNP@C reached the micrometer range by 12 hours incubation at $\mathrm{pH}=3$.

\section{Effect of sodium chloride}

The effect of $\mathrm{NaCl}$ addition on the colloidal stability of our samples is illustrated on Figure 3. The previously mentioned slow aggregation of AgNP@C does not change drastically at $50 \mathrm{mM} \mathrm{NaCl}$ dosage; however, the addition of $150 \mathrm{mM}$ sodium chloride would propagate AgNP aggregation to the micrometer size range, marked by the DLS results (Figure 3A). The drastic changes in aggregation are due to the increase in $\mathrm{Na}^{+}$concentration, as in large quantities these ions can shield the negatively charged surface groups providing electrostatic stabilization. With the decreased repulsion, the particles could form larger aggregates upon collision. After 24 hours, the average hydrodynamic diameter of AgNP@C incubated in a solution containing $150 \mathrm{mM} \mathrm{NaCl}$ decreased, compared to its state after 12 hours of incubation (Figure 3A). This result suggested heavy sedimentation where the aggregates have grown to a size where the DLS instrument could no longer determine the Z-average parameter accurately. The nanosilver sample prepared with green tea showed similar tendencies in Z-average and zeta potential changes, as well as in spectral shifts (Figure 3B), although it is important to note that the attenuating nature of the biological matrix again prevented drastic increases in the average hydrodynamic diameter.

\section{Effects of glucose and glutamine}

During contact with living species, nanoparticles encounter a number of small organic molecules that can affect their surface charge upon adsorption and affect their colloidal stability. Glucose and glutamine are the most prominent members of monosaccharides and free amino acids, respectively; hence, they were selected for the screening experiments. In the presence of glucose, biomolecule interactions with the nanoparticle surface could cause a shift in the characteristic absorbance peak of AgNPs (Figure S3). Similarly, the maximum of the SPR spectra of AgNP@GT is at a longer wavelength than its particle size would indicate, as observed previously (Figure 1B). Despite the UV-Vis shifts, the presence of glucose led to no considerable changes in aggregation grade (determined by DLS measurements) in either AgNP samples (Figure S3).

No notable increase in Z-average values were observed after adding glutamine to the citrate-stabilized nanoparticle colloid system (Figure S4A); however, the peak of the UV-Vis spectrum of AgNP@C clearly shifted to higher wavelength values, most likely due to amino acid adsorption on the nanoparticle surface. ${ }^{29}$ In case of AgNP@GT (Figure S4B), the presence of glutamine caused numerous changes, such as the red shift of the SPR peak in the spectrum, which indicated surface interactions between the particles and amino acid molecules. This idea was supported by the increase in zeta potential from below $-30 \mathrm{mV}$ to around -20 $\mathrm{mV}$ depending on the elapsed incubation time (Figure S4B). ${ }^{44}$ The changes also affected the $Z$-average values, where the average diameter increased 2-3 fold, implying that glutamine molecules interfered with the integrity of the green tea multicomponent matrix. While this increase confirms the interaction between AgNPs and glutamine, the actual decrease in colloidal stability is far less pronounced than the effect of increased sodium chloride concentration.

\section{Effect of cell culture components}

In order to test the nanoparticle aggregation behavior in biological fluids modeling complex environments, AgNP aggregation grade was measured in the presence of FBS both in aqueous conditions and in DMEM. ${ }^{28,29}$ According to our UV-Vis and DLS results (Figures 4 and S5), in case of AgNP@C, DMEM caused quick and large-scale particle aggregation due to its high electrolyte concentrations, with the average aggregate size reaching the micrometer range by the end of 24 hours incubation. On its own, FBS had essentially no effect on hydrodynamic diameter, while it shifted the characteristic UV-Vis absorption peak and decreased zeta potential in the sample (Figure 4A). These observations supported the formation of a biomolecular corona around the particles, similarly to the small molecules like glutamine. We repeated the experiment using a solution containing both DMEM and FBS, where we found decreased aggregation grade and simultaneously a 


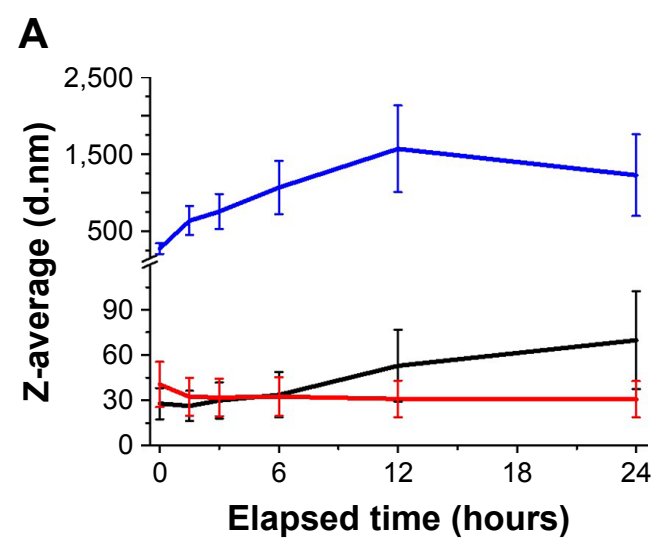

AgNP@C
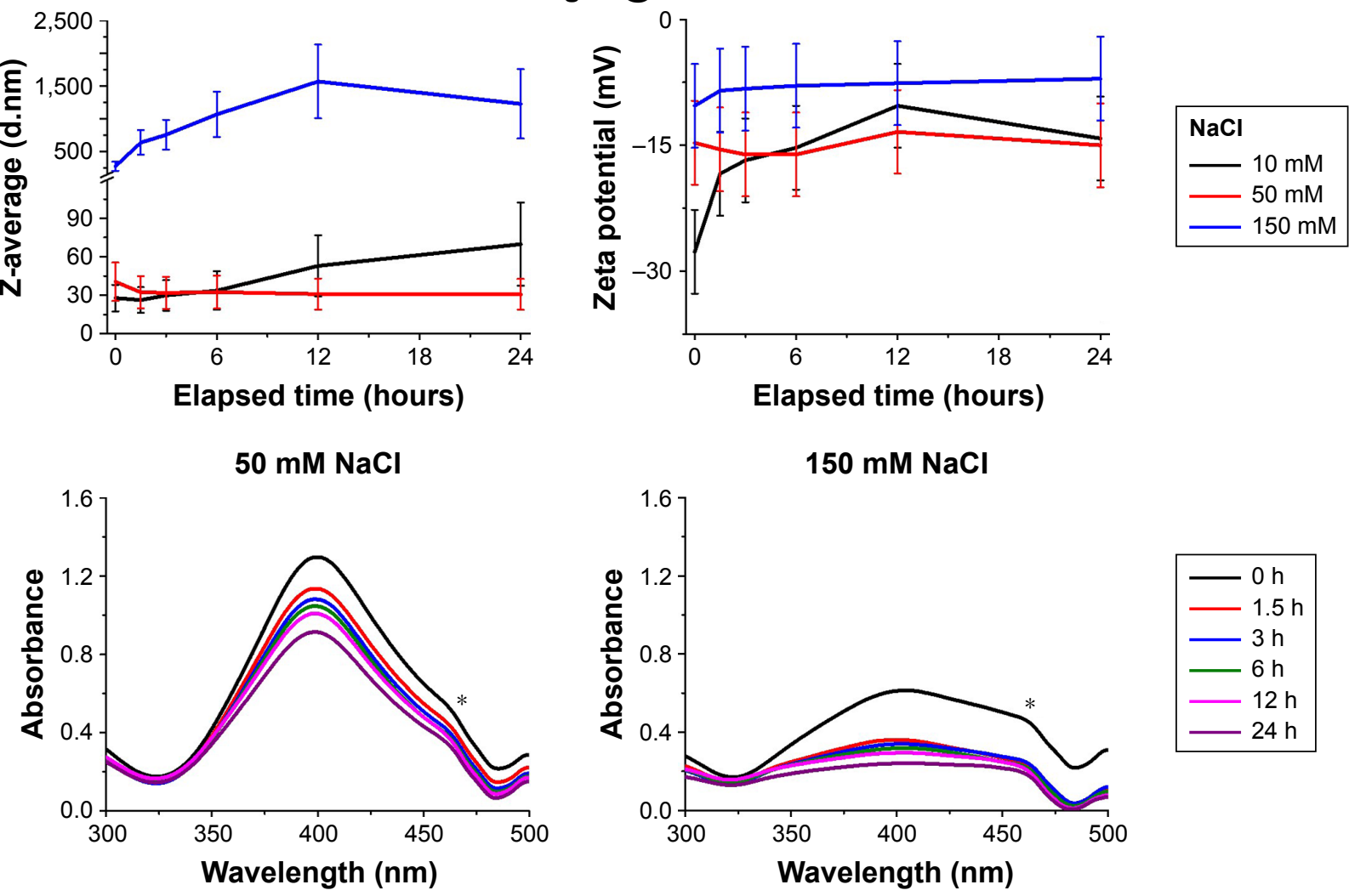

B

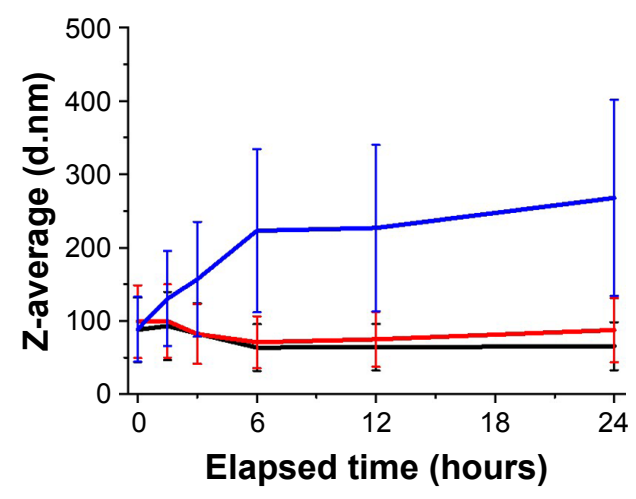

$50 \mathrm{mM} \mathrm{NaCl}$

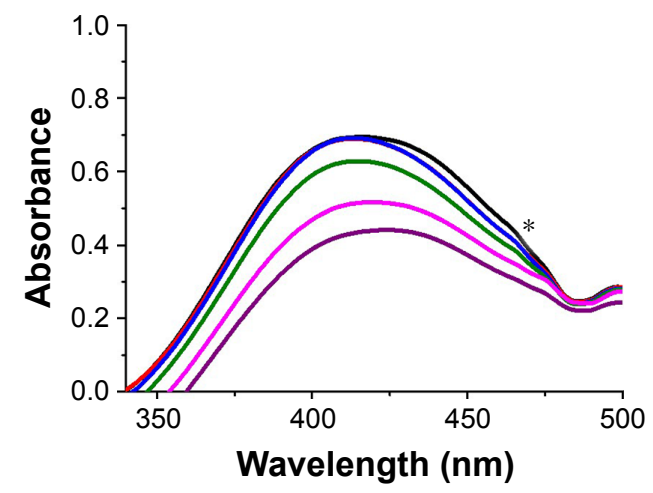

AgNP@GT
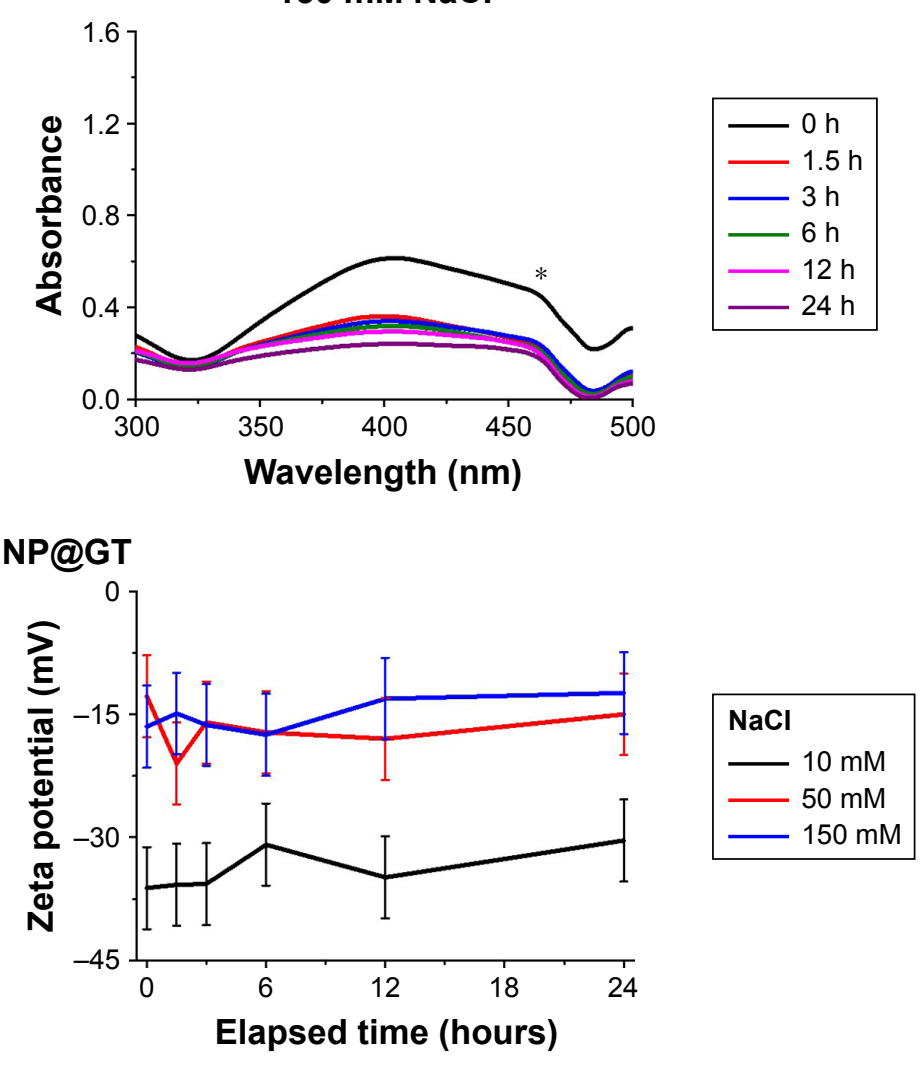
A
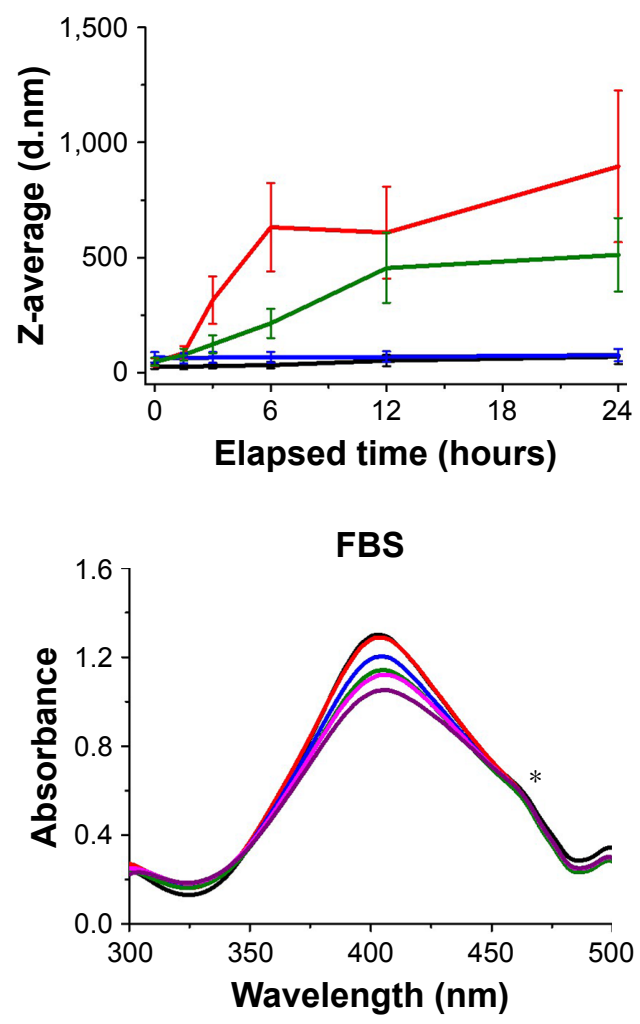

AgNP@C
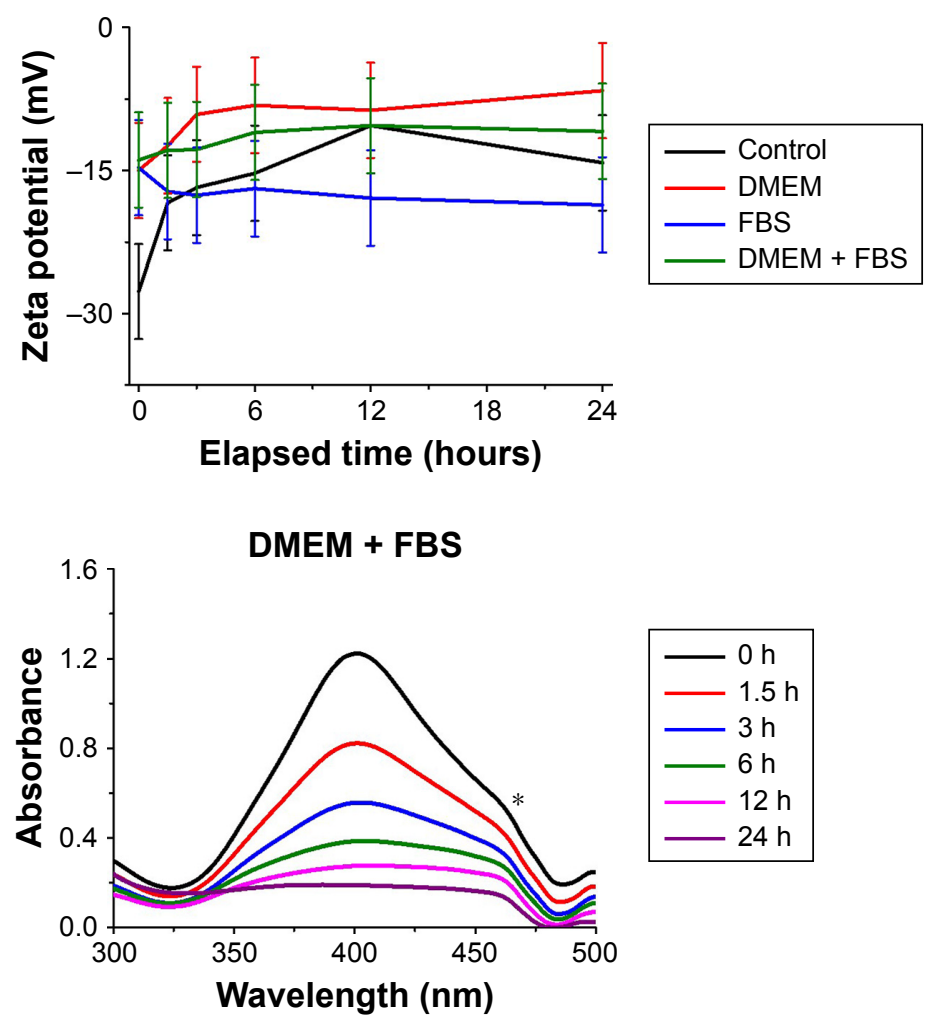

B
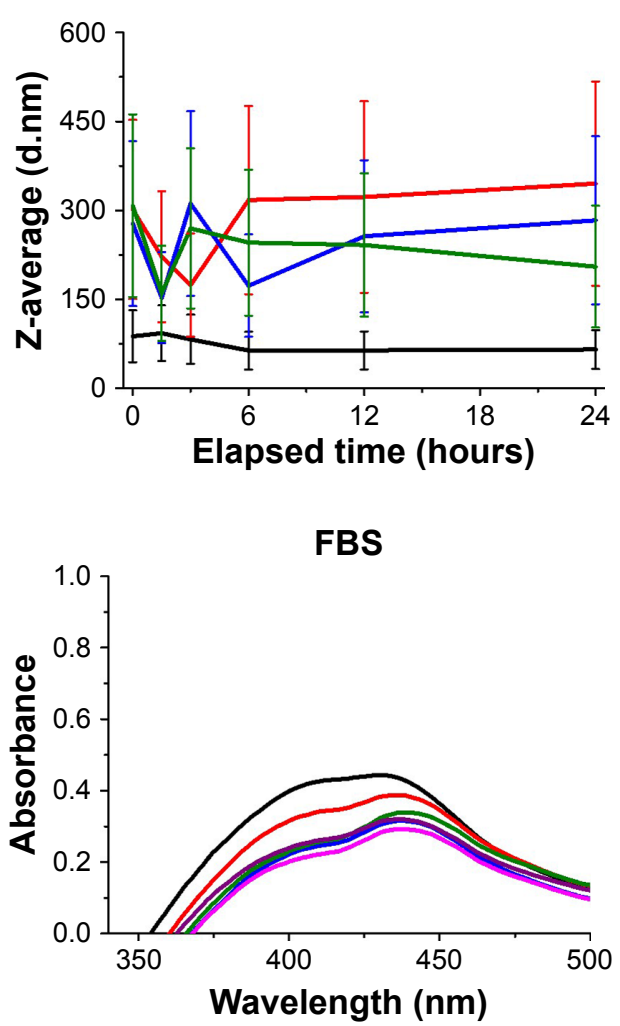

AgNP@GT
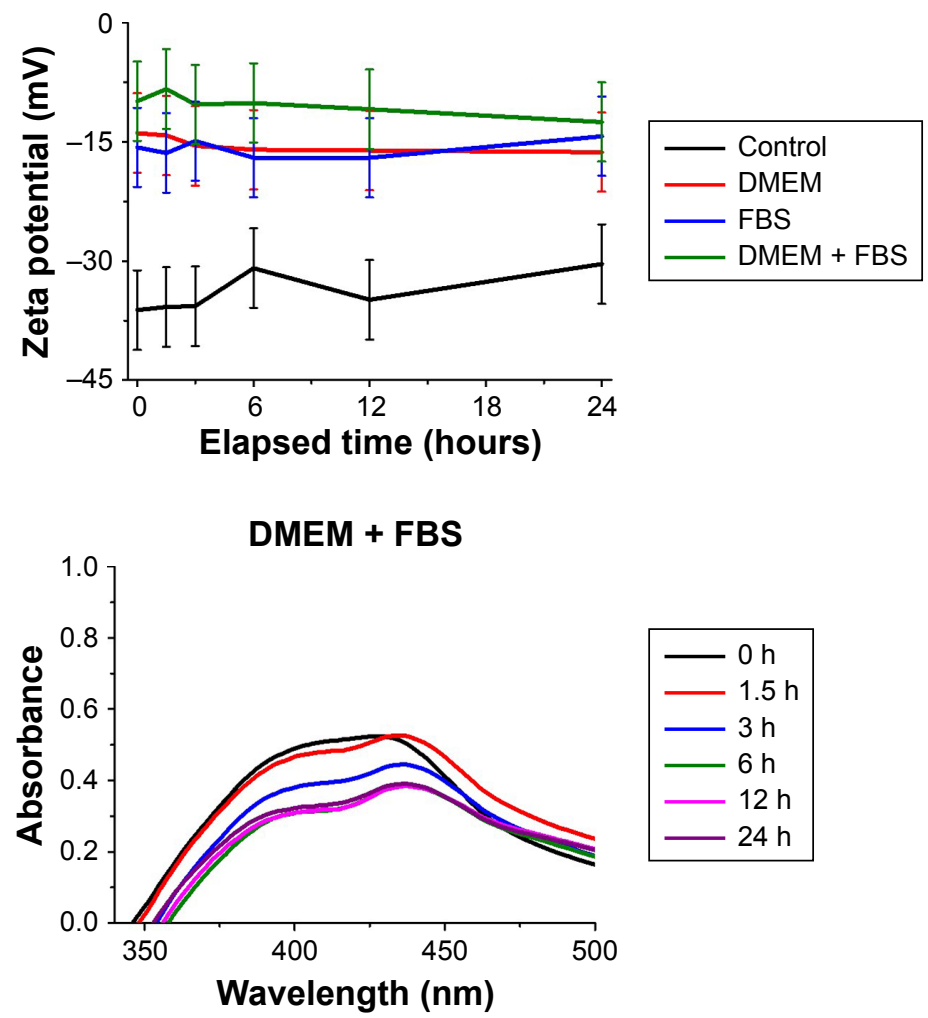

Figure 4 The effect of cell culture medium components DMEM and FBS on the aggregation behavior of the as-prepared silver nanoparticles.

Notes: Average hydrodynamic diameter (Z-average) trend, zeta potential, and UV-Vis spectrum changes of (A) citrate-stabilized AgNP@C, (B) green tea-stabilized AgNP@ GT, observed over 24 hours. * marks a UV-Vis detection error during the measurements that should be disregarded.

Abbreviations: AgNP@C, citrate-capped nanosilver; AgNP@GT, green tea extract-stabilized silver nanoparticle. 
decelerated aggregation process. ${ }^{44}$ According to these results, the biomolecular corona effect could be one of the most important phenomena regarding biological interactions of AgNPs stabilized by small molecules such as citrate.

For the nanosilver sample prepared by green tea extract, both FBS and DMEM increased the aggregation grade separately; however, in the FBS-containing DMEM, the aggregation attenuating effect of FBS was observable (Figure 4B). Our results indicate that biomolecules of the serum interacted with the AgNP@GT particles in a way to improve their stability, probably due to the gradual and dynamic change in the composition of the biomolecular corona surrounding the particles.

\section{Toxicity on human cells}

To evaluate how the cytotoxic propensity can be influenced by nanoparticle aggregation, we performed cell viability assays on human A549 adenocarcinoma as well as on non-cancerous MRC-5 fibroblast cells. The aggregationdependent toxicity of both AgNP@C and AgNP@GT

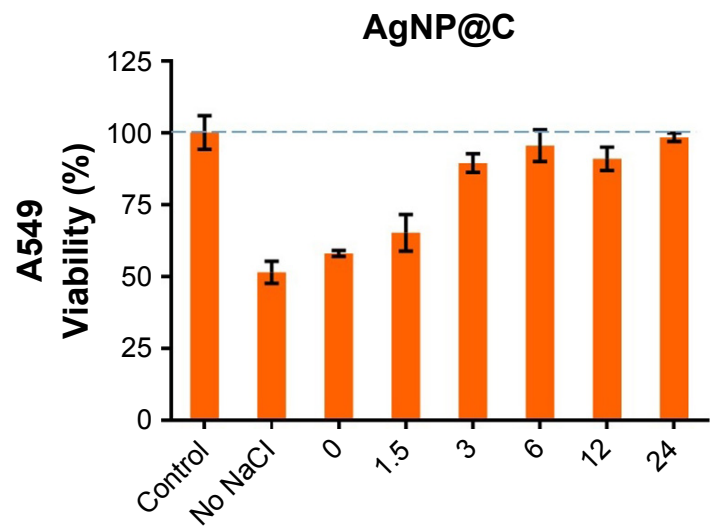

Aggregation time (hours)

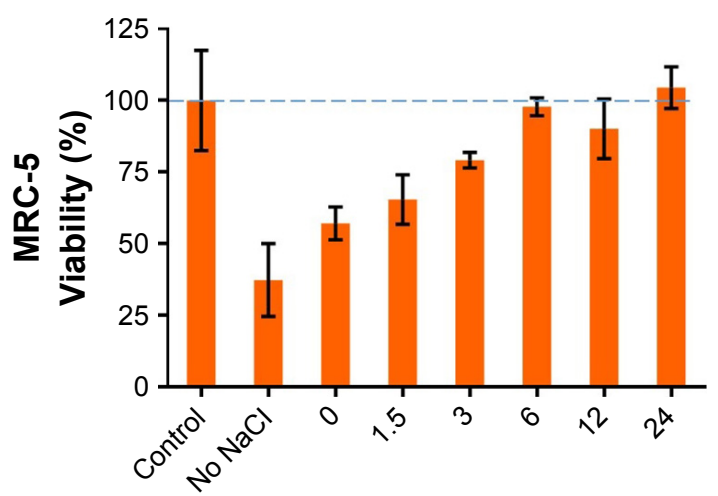

Aggregation time (hours) samples was investigated at nanoparticle concentrations corresponding to the respective cell line-specific $\mathrm{IC}_{50}(\mathrm{~A} 549$ : $\mathrm{IC}_{50 \mathrm{AgNP} @ \mathrm{C}}=72.2 \pm 4.1 \mathrm{ppm} ; \mathrm{IC}_{50 \mathrm{AgNP} @ \mathrm{GT}}=63.1 \pm 3.8 \mathrm{ppm} ;$ MRC-5: $\left.\mathrm{IC}_{50 \mathrm{AgNP} @ \mathrm{C}}=17.6 \pm 3.0 \mathrm{ppm} ; \mathrm{IC}_{50 \mathrm{AgNP} @ \mathrm{GT}}=1.3 \pm 0.2 \mathrm{ppm}\right)$. The $\mathrm{IC}_{50}$ concentrations were determined by MTT assay prior to aggregation-related viability measurements. These MTT experiments were assessed by incubating the cell lines with aggregated AgNP samples for 24 hours. Nanoparticle aggregation states were formulated by incubating AgNP@C and AgNP@GT samples with $150 \mathrm{mM} \mathrm{NaCl}$ for $0,1.5,3$, 6,12 , and 24 hours at $37^{\circ} \mathrm{C}$ prior to the beginning of the toxicity assays, as our results indicated that among the tested experimental conditions the electrolyte concentration had the greatest impact on nanoparticle aggregation.

The MTT viability assays performed on A549 and MRC-5 cell lines revealed that nanoparticle toxicity universally decreased with growing aggregation grade (Figure 5). Cell viability was the lowest $(\sim 50 \%)$ when cells were exposed to AgNPs which were not pre-incubated with $\mathrm{NaCl}$, thus showing the toxic effect of both AgNPs at their $\mathrm{IC}_{50}$ concentrations.

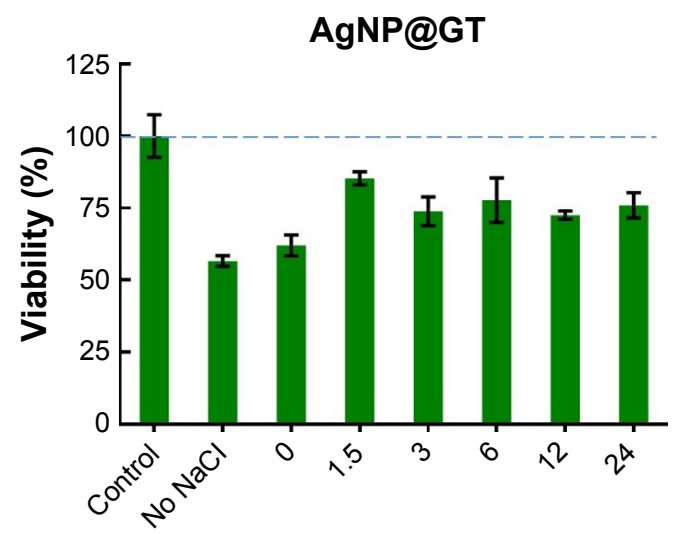

Aggregation time (hours)

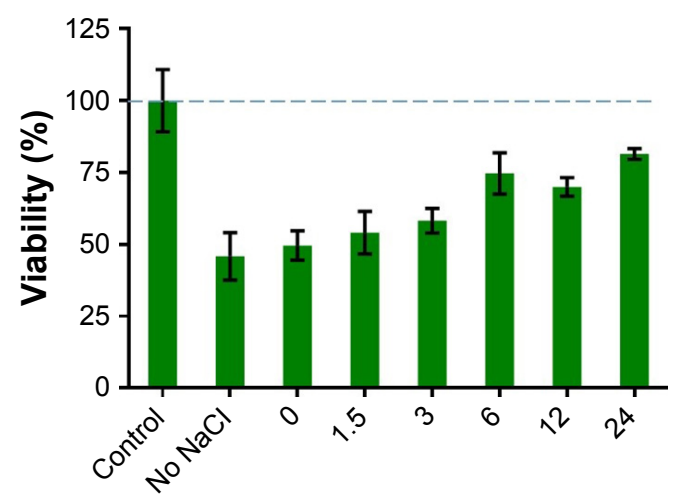

Aggregation time (hours)

Figure 5 The effect of nanoparticle aggregation (citrate-stabilized: AgNP@C, green tea-stabilized: AgNP@GT) on cytotoxicity toward A549 human lung cancer and MRC-5 human fibroblast cells.

Note: Increasing aggregation grades were prepared using $150 \mathrm{mM} \mathrm{NaCl}$ for longer time intervals up to 24 hours.

Abbreviations: AgNP@C, citrate-capped nanosilver; AgNP@GT, green tea extract-stabilized silver nanoparticle. 
The viability of both A549 and MRC-5 cells were higher when they were treated with AgNP samples, which were previously left to aggregate by incubation with $150 \mathrm{mM}$ $\mathrm{NaCl}$ for longer periods of time. Differences between the effects of citrate-capped and green tea extract-stabilized AgNP samples could also be observed, since no toxicity was detected for AgNP@C after 6 hours incubation with $\mathrm{NaCl}$ due to its relatively weak electrostatic stabilization; however, the more stable AgNP@GT retained a small degree of cytotoxic activity for the entire aggregation timeframe ( $\sim 80 \%$ cell viability).

\section{Antimicrobial activity}

Finally, antibacterial and antifungal activities of aggregated AgNP@C and AgNP@GT were tested with the same procedure described in the "Toxicity on human cells" section. The minimal inhibitory concentration of AgNP@GT was lower $(\sim 0.59 \mathrm{ppm})$ against all of the three examined microbial strains than the MIC of AgNP@C ( $\sim 18.75$ ppm for $C$. neoformans and B. megaterium, $\sim 37.5 \mathrm{ppm}$ for E. coli). The inhibitory effect of the aggregated nanoparticles decreased throughout with growing nanoparticle aggregation grade. Microbial cell viability was the lowest in contact with AgNPs without $\mathrm{NaCl}$ pre-incubation, while the viability of all three strains increased when they were treated with AgNP samples previously left to aggregate for different periods of time (Figure 6). No toxicity was detected for AgNP@C after 12 hours incubation with $\mathrm{NaCl}$ for $B$. megaterium and $E$. coli while its effectiveness against $C$. neoformans was lost after 24 hours aggregation with $\mathrm{NaCl}$. AgNP@GT could retain a certain degree of inhibitory activity for the entire aggregation timespan $(\sim 20 \%$ activity for B. megaterium and E. coli and $\sim 50 \%$ for $C$. neoformans).

\section{Discussion}

The production and utilization of AgNPs is a rapidly growing field of industry, which will eventually lead to the enhanced release and accumulation of these particles in the environment. Therefore, there is an ever increasing demand to investigate the behavior, transport, and toxicity of the synthesized AgNPs. As AgNPs have a tendency to aggregate, the kinetics of this aggregation process can be a major factor in governing not only the biological activity of the particles but also their fate in the environment and in contact with various living organisms. Consequently, we aimed to acquire further knowledge on all those circumstances, agents, and parameters that modulate nanoparticle agglomeration in biorelevant conditions that might influence the reactivity of AgNPs on living cells.
The initial objective of our research was to synthesize different AgNP samples with comparable morphologies and sizes, as both of these factors affect optical and biological properties. ${ }^{48}$ Research by Pal et al showed that nanoparticle shape can influence antibacterial activity as different particle shapes propagate different crystallinity and high-atomdensity facets such as $\{111\}$ possess higher reactivities. ${ }^{40}$ Considering particle size, our own previous contributions align with the literature as we observed that smaller AgNPs have higher toxicity compared to larger ones. ${ }^{39}$

Both the chemical reduction and green synthesis methods were capable of forming quasi-spherical nanoparticles with comparable sizes according to our TEM images. However, there were also slight differences between the two applied approaches; while the chemical reduction method allowed us to control most of the parameters affecting NP formation, the method using green tea extract provided less control over key factors, like the ratio and the quality of reducing and stabilizing agents. This resulted in bigger polydispersity and lesser shape specificity in the AgNP@GT sample, which led to a broader characteristic UV-Vis spectrum compared to the one of AgNP@C. Despite these slight differences in shape specificity, the results of our electron diffraction measurements verified that both the samples present the same crystal facets; therefore, similar biological activities were expected which made the subsequent comparative measurements possible.

In this study, we characterized particle aggregation based on DLS and UV-Vis measurements, which showed analogous changes when there were no biomolecular components in the system: the increase of the average hydrodynamic diameter and the decrease in the characteristic SPR values both pointed toward AgNP aggregation. In the presence of biomolecules, however, their interactions with nanoparticle surfaces invoked a red shift in the SPR peaks owing to dielectric constant changes on the NP surfaces, in good agreement with previous reports. ${ }^{29}$ However, it also has to be noted that the peak shifts in the UV-Vis spectra were used only to verify and illustrate NP aggregation and are not expected to be suitable for quantitative analysis, since the green tea extract (the spectrum of which we used as background for AgNP@ GT measurements) possesses $\mathrm{pH}$ indicator capabilities, and its chemical composition inevitably undergoes some mild or moderate changes during $\mathrm{Ag}^{+}$reduction.

Considering the effects of the selected biorelevant conditions, the largest impact on AgNP aggregation was observed by increasing $\mathrm{NaCl}$ concentration followed by the effects of acidic $\mathrm{pH}$, in agreement with previous studies. ${ }^{23-25}$ Nanoparticle surface interactions indicated by SPR changes were detected in the presence of glucose, glutamine, and 


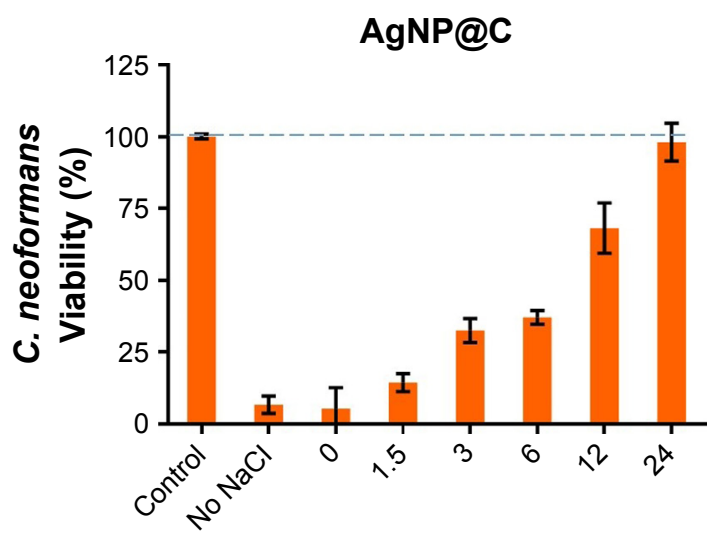

Aggregation time (hours)

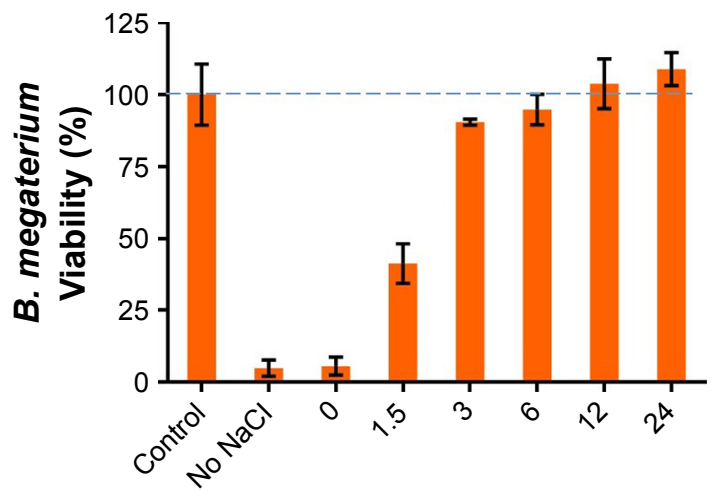

Aggregation time (hours)

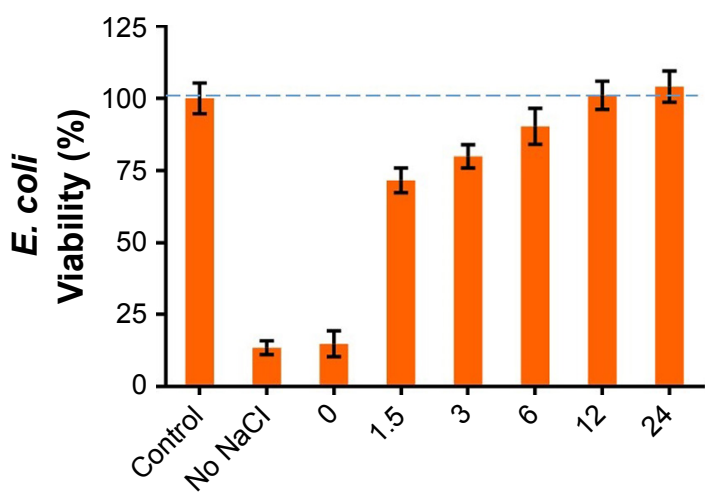

Aggregation time (hours)

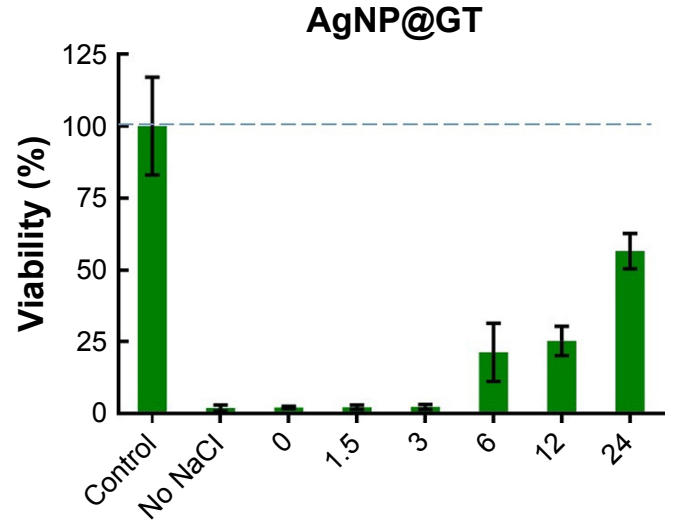

Aggregation time (hours)

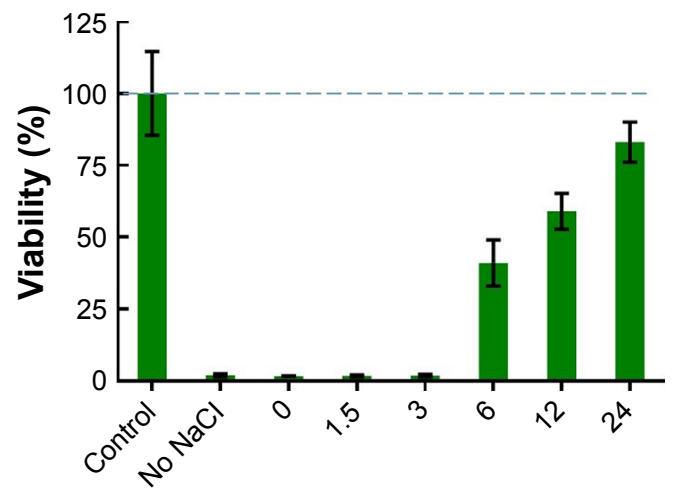

Aggregation time (hours)

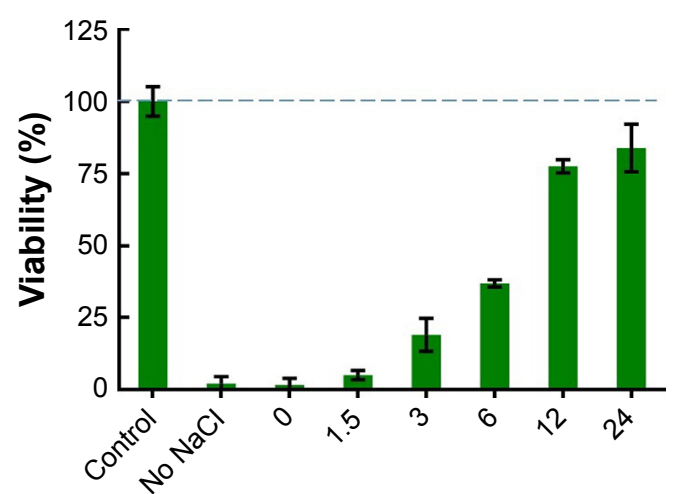

Aggregation time (hours)

Figure 6 The effect of nanoparticle aggregation (citrate-stabilized: AgNP@C, green tea-stabilized: AgNP@GT) on the antimicrobial activity against C. neoformans, B. megaterium, and E. coli.

Note: Increasing aggregation grades were prepared using $150 \mathrm{mM} \mathrm{NaCl}$ for longer time intervals up to 24 hours.

Abbreviations: AgNP@C, citrate-capped nanosilver; AgNP@GT, green tea extract-stabilized silver nanoparticle.

FBS in the nanoparticle colloid solutions similarly to the results obtained when human serum albumin was absorbed on AgNP surfaces in the research of Alarcon et al. ${ }^{29}$ These interactions contributed to the well-known biomolecular corona formation which attenuated the AgNP aggregation grade counteracting the large amount of electrolytes present in DMEM. ${ }^{28,29,44}$

The observed changes in the average hydrodynamic diameter of AgNP@GT in the presence of medium components suggested that while green tea extracts are mostly made up of flavonoids and carbohydrates, the protein-rich composition of FBS offers a greater degree of electrosteric interactions that are able to provide improved colloidal stability compared to GT components. ${ }^{24,25,45}$ In the green tea matrix surrounding AgNP@GT particles, changes happened slowly compared to the citrate-stabilized sample. This allowed us to observe that within both FBS and DMEM + FBS environments, average hydrodynamic diameter values peaked at around 3 hours of 
incubation, and then the values decreased (Figure 4B). This can be explained by the initial formation of a large and less compact biomolecule layer adsorbed on the nanoparticle surface, referred to as "soft corona" by the literature, and its eventual shift to a smaller, more compact state, where the absorbed biomolecules have a higher affinity toward the nanoparticle surface called "hard corona," as it was suggested by Riviere et al, even though more recent and generally accepted studies hypothesize a core-shell-like structure for the corona model. ${ }^{26,27}$ Our results do not contradict the descriptions of these newer corona models, and no further measurements were taken to clearly define the background of this phenomenon, but our results nonetheless seem to indicate a size decrease that could be explained with the slow formation of a more specific and compact biomolecular layer on the particle surfaces.

The aggregation degree of AgNPs is dependent on a variety of different conditions, and it is necessary to examine these parameters on systems that closely resemble living organisms. However, based on our measurements, it can be presumed that the results involving the less convenient and more expensive cell culture components can be closely estimated by the simpler approaches we utilized. The results of the $150 \mathrm{mM} \mathrm{NaCl}$ measurements closely resemble the ones of the experiments involving DMEM, while the results associated with the presence of glutamine are fairly similar to the data obtained with FBS.

Our comprehensive biological screening concluded that AgNP toxicity decreases with increasing aggregation grade; hence, it emphasizes the massive impact of the corona effect on the biological activity of nanoparticles especially in biomedical applications.

Comparative analysis of the data obtained on citrate and green tea extract-stabilized AgNP samples also delivered some interesting results. In good agreement with previous findings, the electrostatic stabilization provided by the citrate groups of AgNP@C resulted in weaker colloidal stability compared to that of AgNP@GT, where the electrosteric nature of the green tea extract offers greater protection against aggregation. ${ }^{24,25}$ The importance of this difference was especially pronounced during the biological screenings, where we observed that AgNP@C lost its cytotoxic and antimicrobial activity within 6 hours of aggregation (with the exception of C. neoformans, where it took 24 hours to reach complete loss of effect, since this strain showed higher sensitivity toward silver), while AgNP@GT retained its toxicity, albeit showing a weaker toxic feature.

Thus our results indicate that the appropriate stabilizing mechanism of AgNP colloids via well-selected capping agents has a profound effect on their aggregation propensity and biological activity. Particle aggregation is closely associated with the chemistry of the capping agents; furthermore, it is a function of $\mathrm{pH}$, ionic strength, and electrolyte composition, since all of them are key variables to the behavior of AgNPs both in natural and in engineered environments.

While there have been previous contributions investigating NP colloidal stability as the function of environmental factors, ${ }^{23-25}$ or AgNP surface interactions with biomolecules, ${ }^{26,27,29}$ to our knowledge this is the first time that a systematic examination was performed on the aggregation behavior of differently synthesized AgNPs with such a wide variety of parameters while simultaneously measuring the effect of aggregation on biological activity on both human cells and microbes. Based on our results, electrostatically stabilized silver nanomaterials might not be suitable for biomedical applications in their initial form. On the other hand, green approaches offer a new frontier, since next to their environmentally benign nature they also provide protection from large-scale aggregation, particularly under such conditions that are typical in living organisms. Besides optimization of green synthesis methods, subsequent steps toward broad range utilizations should consider the active exploitation of the biological matrix surrounding the particles, by the controlled formation of an active, rationally designed biomolecular corona which assigns synergistic or targeting features to the nanoparticles.

\section{Conclusion}

In order to routinely and reliably use AgNPs for any therapeutic or environmental application, it is required to consider the colloidal stability of these materials in biological environments, as aggregation can influence key properties within nanoscale systems. To our knowledge, this is the first time that a systematic examination on AgNP aggregation behavior was performed with a large variety of parameters while simultaneously measuring the effect of aggregation on biological activity. Our results indicate that nanoparticle colloidal stability in more complex systems, like complete cell culture media, can be closely estimated by simpler model compounds like sodium chloride, glucose, or glutamine. It can also be assumed based on our findings that purely electrostatic stabilization might not be suitable for biomedical AgNP applications, since AgNPs stabilized by citrate groups undergo large-scale aggregation near physiological electrolyte concentration, resulting in complete loss of their biological activity. On the other hand, green synthesis methods could offer a new dimension in medical applications, 
as the green tea extract could attenuate aggregation grade in AgNP@GT due to electrosteric interactions even in complex cell culture media, thus AgNPs prepared by this method retain a certain degree of their desired toxicity. This research emphasizes the vital importance of nanoparticle synthesis and stabilization methods as they have a profound influence on the colloidal stability of the resulting nanoparticles which is a key factor affecting their biological activity.

\section{Acknowledgments}

This work was partially financed by the Hungarian National Research, Development and Innovation Office-NKFIH through grants GINOP 2.3.2-15-2016-00035 and GINOP2.3.2-15-2016-00013 and by the Hungarian Scientific Research Fund through grant OTKA K120115 (ZK).

\section{Author contributions}

All authors contributed to data analysis, drafting or revising the article, gave final approval of the version to be published, and agree to be accountable for all aspects of the work.

\section{Disclosure}

The authors report no conflicts of interest in this work.

\section{References}

1. Saji VS, Choe HC, Yeung KWK. Nanotechnology in biomedical applications: a review. Int J Nano Biomater. 2010;3(2):119-139.

2. Thota S, Crans DC. Metal Nanoparticles: Synthesis and Applications in Pharmaceutical Sciences. Weinheim, Germany: WILEY-VCH Verlag; 2018.

3. Rai M, Yadav A, Gade A. Silver nanoparticles as a new generation of antimicrobials. Biotechnol Adv. 2009;27(1):76-83.

4. Tran QH, Nguyen VQ, Le A-T. Silver nanoparticles: synthesis, properties, toxicology, applications and perspectives. Adv Nat Sci-Nanosci. 2013; 4(3):033001.

5. Jeyaraj M, Sathishkumar G, Sivanandhan G, et al. Biogenic silver nanoparticles for cancer treatment: an experimental report. Colloids Surf B Biointerfaces. 2013;106:86-92.

6. Govender R, Phulukdaree A, Gengan RM, Anand K, Chuturgoon AA. Silver nanoparticles of Albizia adianthifolia: the induction of apoptosis in human lung carcinoma cell line. J Nanobiotechnology. 2013;11(5):5.

7. Huang T, Xu XN. Synthesis and characterization of tunable rainbow colored colloidal silver nanoparticles using single-nanoparticle plasmonic microscopy and spectroscopy. J Mater Chem. 2010;20(44):9867-9876.

8. Xu R, Wang D, Zhang J, Li Y. Shape-dependent catalytic activity of silver nanoparticles for the oxidation of styrene. Chem Asian J. 2006; 1(6):888-893.

9. Syafiuddin A, Salmiati S, Salim MR, Beng Hong Kueh A, Hadibarata T, Nur H. A review of silver nanoparticles: research trends, global consumption, synthesis, properties, and future challenges. J Chin Chem Soc. 2017;64(7):732-756

10. Siddiqi KS, Husen A, Rao RAK. A review on biosynthesis of silver nanoparticles and their biocidal properties. J Nanobiotechnology. 2018;16(1):14.

11. Iravani S, Korbekandi H, Mirmohammadi SV, Zolfaghari B. Synthesis of silver nanoparticles: chemical, physical and biological methods. Res Pharm Sci. 2014;9(6):385-406.
12. Kharissova OV, Dias HV, Kharisov BI, Pérez BO, Pérez VM. The greener synthesis of nanoparticles. Trends Biotechnol. 2013;31(4):240-248.

13. Janardhanan R, Karuppaiah M, Hebalkar N, Rao TN. Synthesis and surface chemistry of nano silver particles. Polyhedron. 2009;28(12): $2522-2530$.

14. Boroumand Moghaddam A, Namvar F, Moniri M, Md Tahir P, Azizi S, Mohamad R. Nanoparticles biosynthesized by fungi and yeast: a review of their preparation, properties, and medical applications. Molecules. 2015;20(9):16540-16565.

15. Kozma G, Rónavári A, Kónya Z, Kukovecz Ákos. Environmentally benign synthesis methods of zero-valent iron nanoparticles. ACS Sustain Chem Eng. 2016;4(1):291-297.

16. Packer L. Handbook of Antioxidants. 2nd ed. Cadenas E, Packer L, editors. New York, NY, USA: Marcel Dekker; 2001.

17. Mekonnen A, Atlabachew M, Kassie B. Investigation of antioxidant and antimicrobial activities of Euclea schimperi leaf extracts. Chem Biol Technol Agric. 2018;5(1):1-12.

18. Saha J, Begum A, Mukherjee A, Kumar S. A novel green synthesis of silver nanoparticles and their catalytic action in reduction of Methylene Blue dye. Sustain Env Res. 2017;27(5):245-250.

19. Jha M, Shimpi NG. Green synthesis of zero valent colloidal nanosilver targeting A549 lung cancer cell: in vitro cytotoxicity. J Genet Eng Biotechnol. 2018;16(1):115-124.

20. Begum NA, Mondal S, Basu S, Laskar RA, Mandal D. Biogenic synthesis of $\mathrm{Au}$ and $\mathrm{Ag}$ nanoparticles using aqueous solutions of Black Tea leaf extracts. Colloids Surf B Biointerfaces. 2009;71(1):113-118.

21. Loo YY, Chieng BW, Nishibuchi M, Radu S. Synthesis of silver nanoparticles by using tea leaf extract from Camellia sinensis. Int $J$ Nanomedicine. 2012;7:4263-4267.

22. Nune SK, Chanda N, Shukla R, et al. Green nanotechnology from tea: phytochemicals in tea as building blocks for production of biocompatible gold nanoparticles. J Mater Chem. 2009;19(19):2912-2920.

23. Elzey S, Grassian VH. Agglomeration, isolation and dissolution of commercially manufactured silver nanoparticles in aqueous environments. J Nanopart Res. 2010;12(5):1945-1958.

24. El Badawy AM, Luxton TP, Silva RG, Scheckel KG, Suidan MT, Tolaymat TM. Impact of environmental conditions $(\mathrm{pH}$, ionic strength, and electrolyte type) on the surface charge and aggregation of silver nanoparticles suspensions. Environ Sci Technol. 2010;44(4):1260-1266.

25. El Badawy AM, Scheckel KG, Suidan M, Tolaymat T. The impact of stabilization mechanism on the aggregation kinetics of silver nanoparticles. Sci Total Environ. 2012;429:325-331.

26. Riviere JE, Scoglio C, Sahneh FD, Monteiro-Riviere NA. Computational approaches and metrics required for formulating biologically realistic nanomaterial pharmacokinetic models. Comput Sci Discov. 2013; 6(1):014005.

27. Hadjidemetriou M, Kostarelos K. Nanomedicine: evolution of the nanoparticle corona. Nat Nanotechnol. 2017;12(4):288-290.

28. Tai JT, Lai CS, Ho HC, et al. Protein-silver nanoparticle interactions to colloidal stability in acidic environments. Langmuir. 2014;30(43): 12755-12764.

29. Alarcon EI, Bueno-Alejo CJ, Noel CW, et al. Human serum albumin as protecting agent of silver nanoparticles: role of the protein conformation and amine groups in the nanoparticle stabilization. J Nanopar Res. 2013;15(1).

30. Rónavári A, Kovács D, Igaz N, et al. Biological activity of greensynthesized silver nanoparticles depends on the applied natural extracts: a comprehensive study. Int J Nanomedicine. 2017;12:871-883.

31. Rónavári A, Igaz N, Gopisetty MK, et al. Biosynthesized silver and gold nanoparticles are potent antimycotics against opportunistic pathogenic yeasts and dermatophytes. Int J Nanomed. 2018;13:695-703.

32. Wan Y, Guo Z, Jiang X, et al. Quasi-spherical silver nanoparticles: aqueous synthesis and size control by the seed-mediated Lee-Meisel method. J Colloid Interface Sci. 2013;394:263-268.

33. Schneider CA, Rasband WS, Eliceiri KW. NIH Image to ImageJ: 25 years of image analysis. Nat Methods. 2012;9(7):671-675. 
34. Schindelin J, Arganda-Carreras I, Frise E, et al. Fiji: an open-source platform for biological-image analysis. Nat Methods. 2012;9(7):676-682.

35. Kararli TT. Comparison of the gastrointestinal anatomy, physiology, and biochemistry of humans and commonly used laboratory animals. Biopharm Drug Dispos. 1995;16(5):351-380.

36. Diabetes Control and Complications Trial Research Group, Nathan DM, Genuth S, et al. The effect of intensive treatment of diabetes on the development and progression of long-term complications in insulindependent diabetes mellitus. N Engl J Med. 1993;329(14):977-986.

37. Eichelberger L, Water RRB. Nitroden and electrolyte concentration in the brain. J Biol Chem. 1944;154:21-29.

38. Sigma-Aldrich. Dulbecco's Modified Eagle's Medium Product Information. Available from: https://www.sigmaaldrich.com/content/ dam/sigma-aldrich/docs/Sigma/Formulation/d5796for.pdf. Accessed January 17, 2018.

39. Kovács D, Igaz N, Keskeny C, et al. Silver nanoparticles defeat p53-positive and p53-negative osteosarcoma cells by triggering mitochondrial stress and apoptosis. Sci Rep. 2016;6:27902.

40. Pal S, Tak YK, Song JM. Does the antibacterial activity of silver nanoparticles depend on the shape of the nanoparticle? A study of the Gramnegative bacterium Escherichia coli. Appl Environ Microbiol. 2007; 73(6):1712-1720.
41. Hatchett DW, White HS. Electrochemistry of sulfur adlayers on the low-index faces of silver. J Phys Chem. 1996;100(23):9854-9859.

42. Li S, Shen Y, Xie A, et al. Green synthesis of silver nanoparticles using Capsicum annuum L. extract. Green Chem. 2007;9(8):852-858.

43. Vigneshwaran N, Nachane RP, Balasubramanya RH, Varadarajan PV. A novel one-pot "green" synthesis of stable silver nanoparticles using soluble starch. Carbohydr Res. 2006;341(12):2012-2018.

44. Ravindran A, Singh A, Raichur AM, Chandrasekaran N, Mukherjee A. Studies on interaction of colloidal Ag nanoparticles with Bovine Serum Albumin (BSA). Colloids Surf B Biointerfaces. 2010;76(1):32-37.

45. Graham HN. Green tea composition, consumption, and polyphenol chemistry. Prev Med. 1992;21(3):334-350.

46. Avnir D. Recent progress in the study of molecularly doped metals. Adv Mater. 2018;30(41):1706804.

47. Naor H, Avnir D. Electroless functionalization of silver films by its molecular doping. ACS Appl Mater Interfaces. 2015;7(48):26461-26469.

48. Vasireddy R, Paul R, Mitra AK. Green synthesis of silver nanoparticles and the study of optical properties. Nanomater Nanotechno. 2012; $2(2): 8$. 


\section{Supplementary materials}

Investigation of the exact interactions

between green tea components and

\section{nanosilver}

Understanding the relationship between the components of green tea and the prepared silver nanoparticles is crucial, as it can significantly alter the properties of the material. In a recent study, Avnir et al offered a new methodology for molecularly doped metallic materials. ${ }^{1,2}$ The study demonstrated that the differences between 2D arrangement and 3D entrapment of dopant particles toward metals can deeply affect certain properties. For example, poly(dimethylsiloxane) doped iron exhibited better corrosion resistance and doped gallium showed better mechanical properties. The study showed that distinguishing between the 2D and 3D structures is possible with Raman spectroscopy; therefore, measurements were carried out on a Senterra II Raman microscope (Bruker, Billerica, MA, USA) on $532 \mathrm{~nm}$ excitation wavelength, $12.5 \mathrm{~mW}$ laser power, and $4 \mathrm{~cm}^{-1}$ spectral resolution.

In line with previous studies, citrate-capped silver nanoparticle samples like AgNP@C did not exhibit strong Raman activity, with only two subtle peaks present around 1,400 and $1,640 \mathrm{~cm}^{-1}$ (Figure S1). ${ }^{3}$ The components of the green tea extract showed fluorescence with increasing intensity toward higher wavenumbers, and this effect got stronger in the surface-enhanced Raman spectra (SERS) of AgNP@ GT. In the contributions of Avnir et al, it has been stated that successful 3D entrapment is accompanied by peak shifts and broadenings in the Raman spectra of the materials. In order to verify whether the silver sample prepared with the use of green tea produced molecularly doped materials, its Raman spectra was compared with a mixture (AgNP@C + GT) that was prepared with the mixing of as-prepared AgNP@C with green tea extract in the same ratio as described for the synthesis of AgNP@GT. We speculated that since the interactions between the citrate-capped particles and green tea components can only be described with the adsorption of organic molecules on the nanoparticle surfaces, the Raman spectra of this sample would show shifted, narrower, and more distinct peaks compared to AgNP@GT if it is indeed a molecularly doped material. The Raman spectra comparison of these two samples on the right hand side of Figure S1 on the other hand exhibit the same peaks without broadenings. The different intensities can be contributed to the fact that the exact amount and composition of the green tea biomolecules adsorbed on the AgNP surfaces that contribute to the SERS signal is random and that the measurements were carried out in liquid samples and therefore particles with different corona compositions could reach the focal point of the microscope due to diffusion. As both spectra showed the same peaks without shifts or broadenings, the interactions between organic components and nanoparticles should be identical; therefore, we came to the conclusion that AgNP@GT is not a molecularly doped material and the components of green tea are only adsorbed on the particle surfaces.

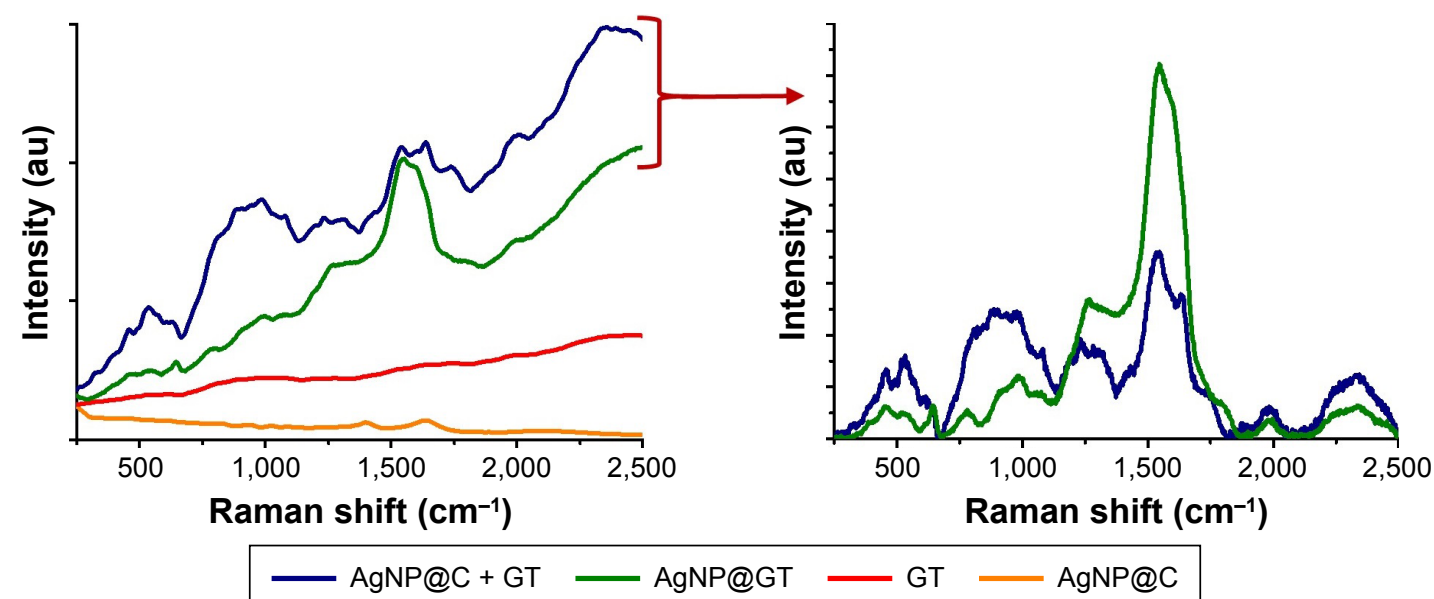

Figure SI Left: Raman spectra of the prepared silver nanoparticle samples (citrate-stabilized AgNP@C, green tea-stabilized AgNP@GT), green tea extract (GT) and citrate stabilized silver nanoparticles mixed with green tea (AgNP@C+GT). Right: Baselined comparison of the Raman spectra of AgNP@GT and AgNP@C+GT.

Abbreviations: AgNP@C, citrate-capped nanosilver; AgNP@GT, green tea extract-stabilized silver nanoparticle. 

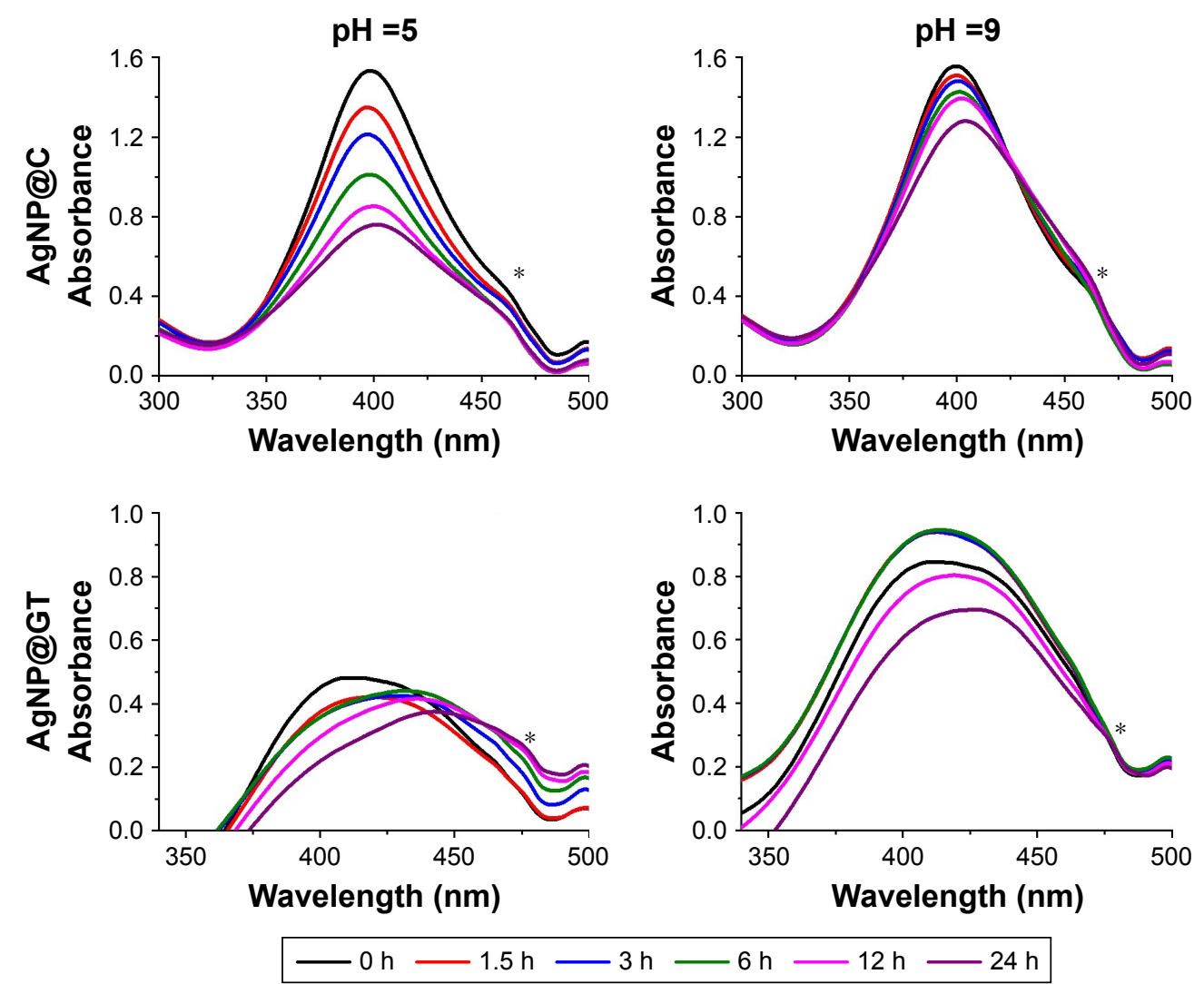

Figure S2 UV-Vis spectral changes of the as-prepared silver nanoparticles (citrate-stabilized AgNP@C, green tea-stabilized AgNP@GT) on pH=5 and pH=9 with 10 mM $\mathrm{NaCl}$ background concentration. * marks a UV-Vis detection error during the measurements that should be disregarded.

Abbreviations: AgNP@C, citrate-capped nanosilver; AgNP@GT, green tea extract-stabilized silver nanoparticle.

A

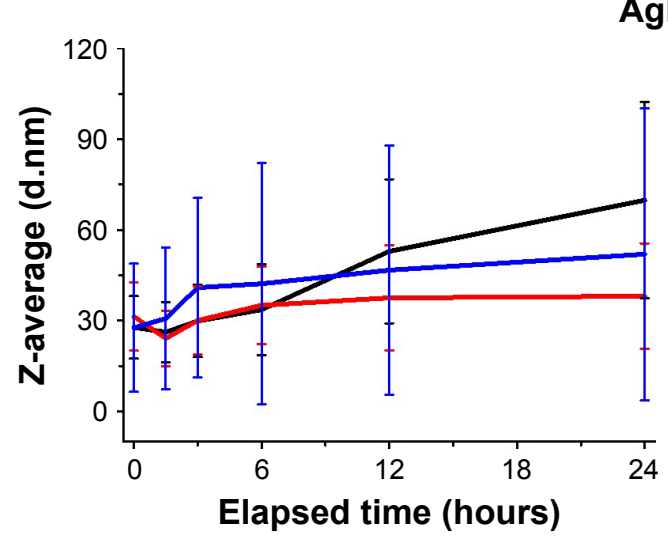

AgNP@C

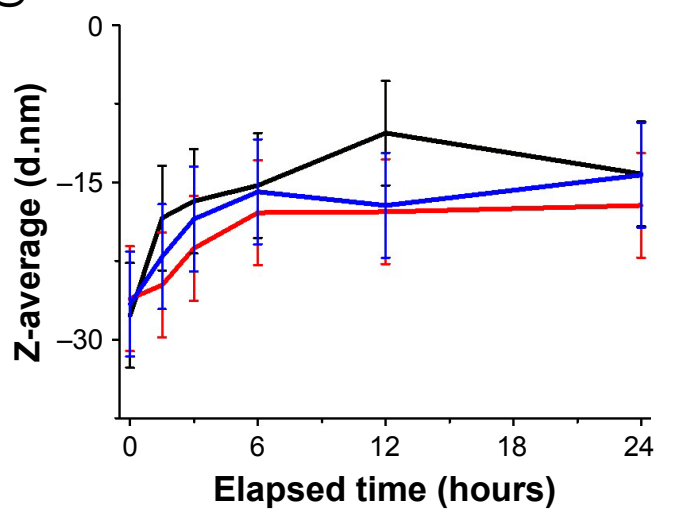

$$
\begin{array}{|lll}
\hline \text { Glucose } & & \\
-0 \mathrm{mM} & -3.9 \mathrm{mM} & -6.7 \mathrm{mM} \\
\hline
\end{array}
$$

Figure S3 (Continued) 

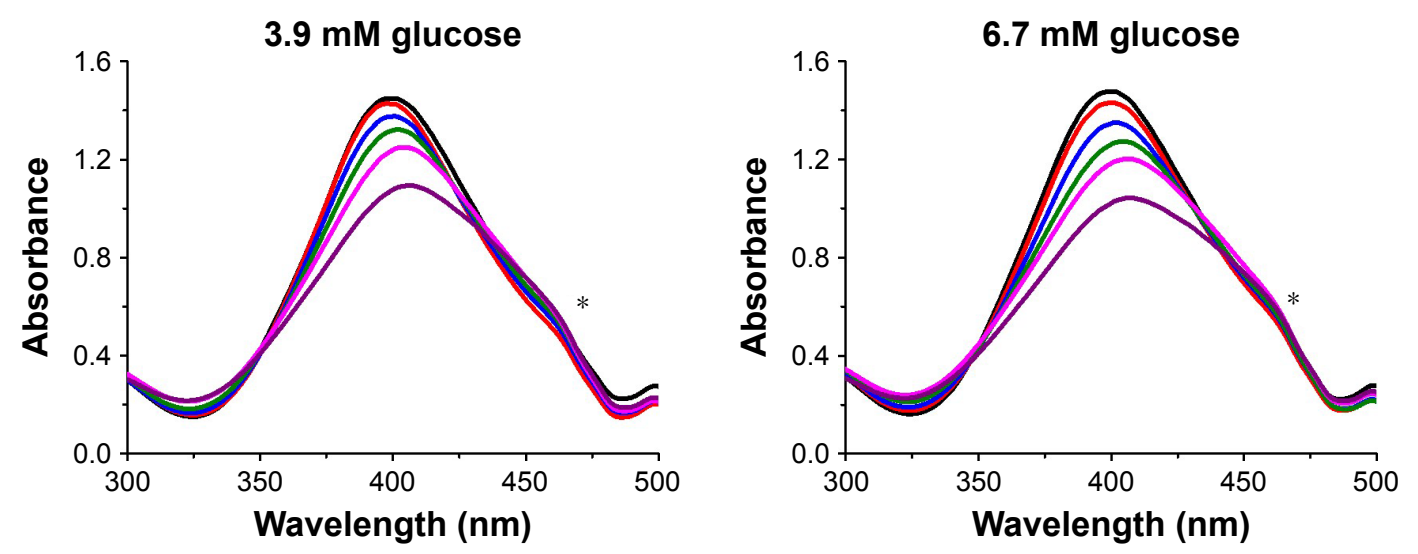

$-0 \mathrm{~h}-1.5 \mathrm{~h}-3 \mathrm{~h}-6 \mathrm{~h}-12 \mathrm{~h}-24 \mathrm{~h}$

B

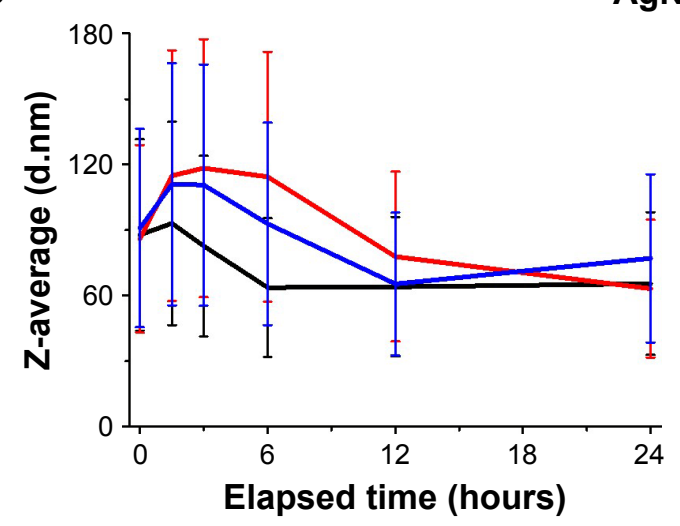

AgNP@GT

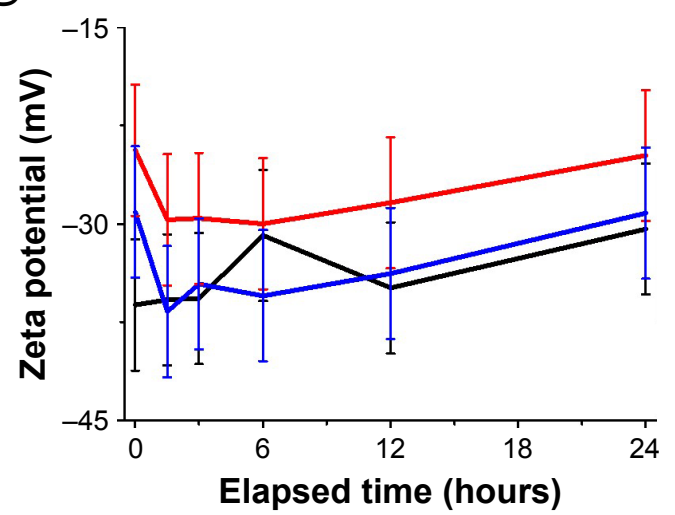

$-0 \mathrm{mM}-3.9 \mathrm{mM}-6.7 \mathrm{mM}$
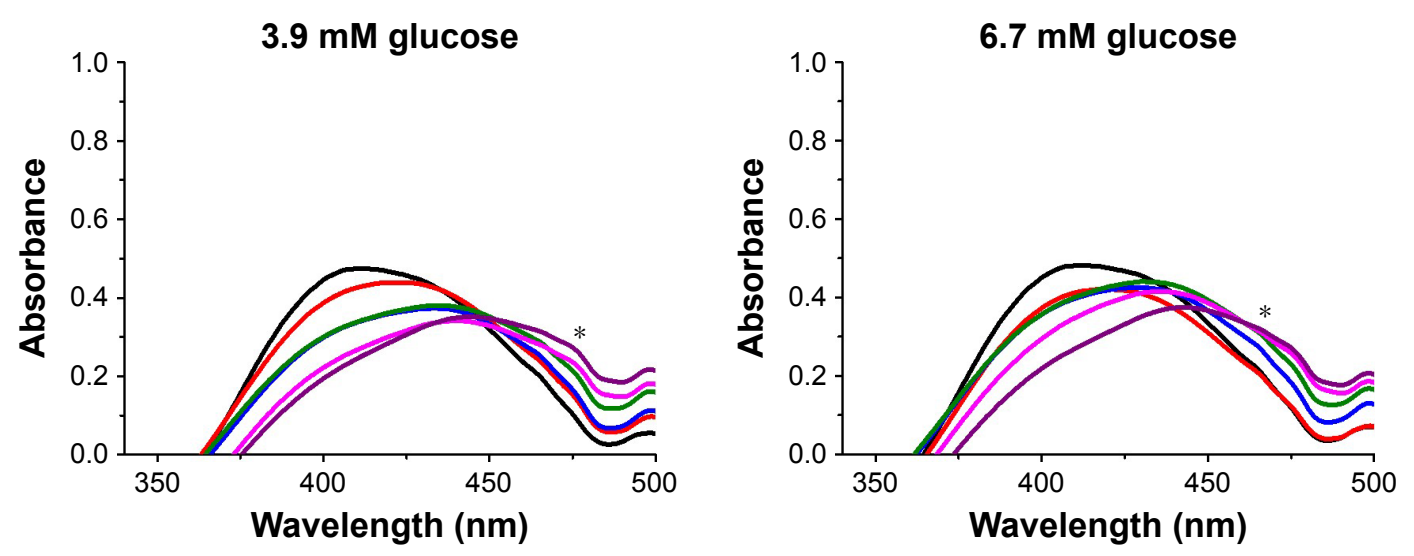

$-0 \mathrm{~h}-1.5 \mathrm{~h}-3 \mathrm{~h}-6 \mathrm{~h}-12 \mathrm{~h}-24 \mathrm{~h}$

Figure S3 The effect of glucose on the aggregation behavior of the as-prepared silver nanoparticles with $10 \mathrm{mM} \mathrm{NaCl}$ background concentration on $\mathrm{pH} \sim 7.2$. Average hydrodynamic diameter (Z-average) trend, zeta potential, and UV-Vis spectrum changes of $(\mathbf{A})$ citrate-stabilized AgNP@C, (B) green tea-stabilized AgNP@GT, observed over 24 hours. * marks a UV-Vis detection error during the measurements that should be disregarded.

Abbreviations: AgNP@C, citrate-capped nanosilver; AgNP@GT, green tea extract-stabilized silver nanoparticle. 


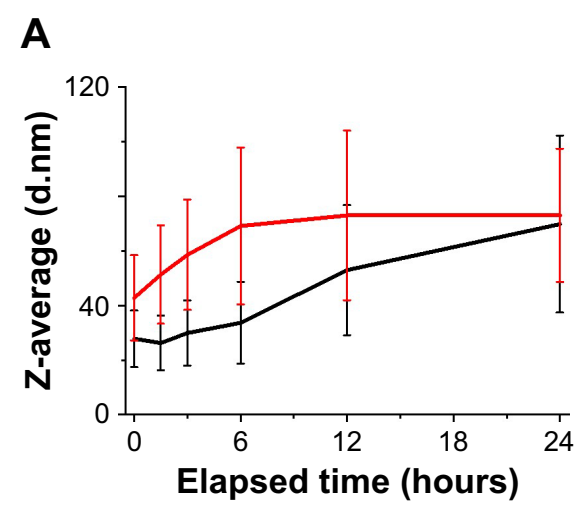

AgNP@C

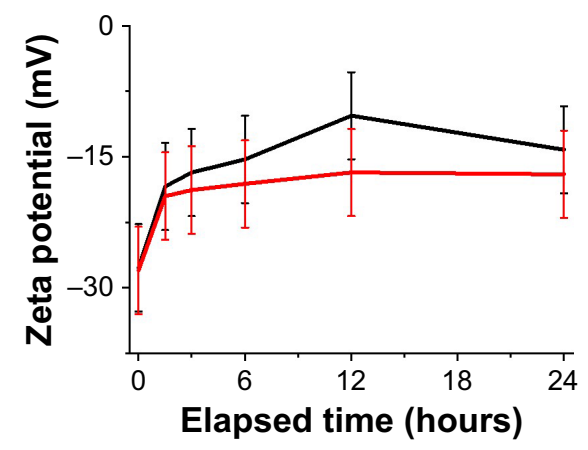

\begin{tabular}{|l|} 
Glutamine \\
$-0 \mathrm{mM}-4 \mathrm{mM}$ \\
\hline
\end{tabular}

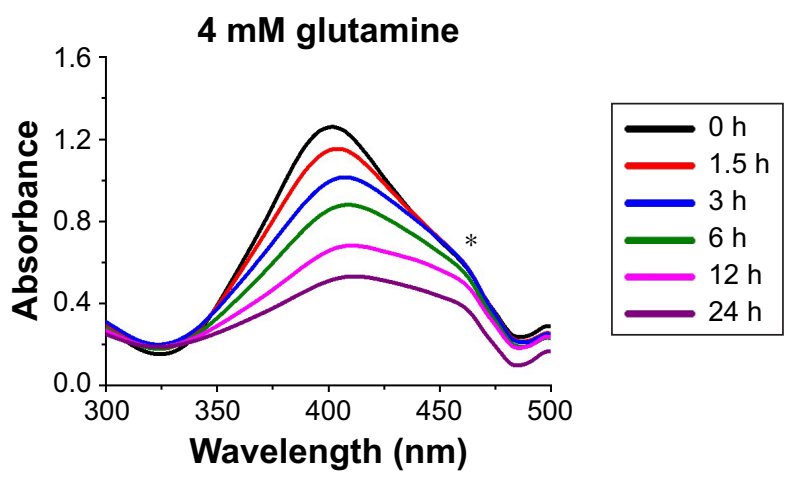

B

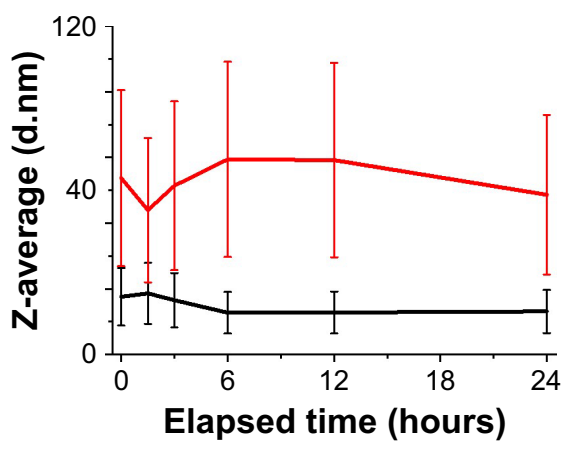

AgNP@GT

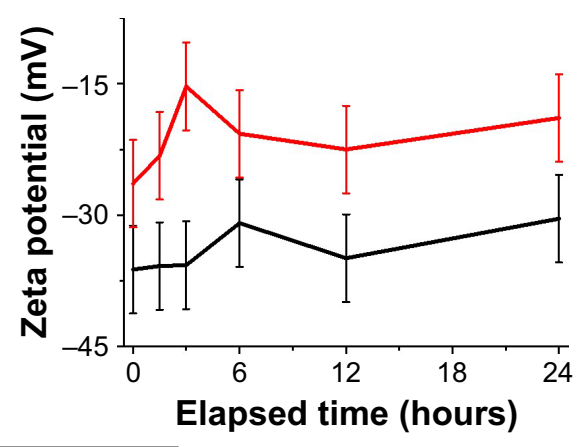

Glutamine
$-0 \mathrm{mM}-4 \mathrm{mM}$

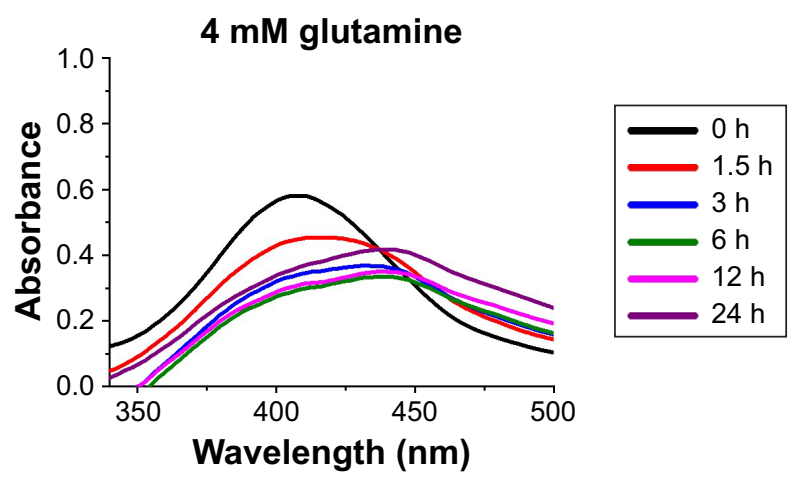

Figure S4 The effect of glutamine on the aggregation behavior of the as-prepared silver nanoparticles with $10 \mathrm{mM} \mathrm{NaCl}$ background concentration on pH 7.2. Average hydrodynamic diameter (Z-average) trend, zeta potential, and UV-Vis spectrum changes of (A) citrate-stabilized AgNP@C, (B) green tea-stabilized AgNP@GT, observed over 24 hours. * marks a UV-Vis detection error during the measurements that should be disregarded.

Abbreviations: AgNP@C, citrate-capped nanosilver; AgNP@GT, green tea extract-stabilized silver nanoparticle. 

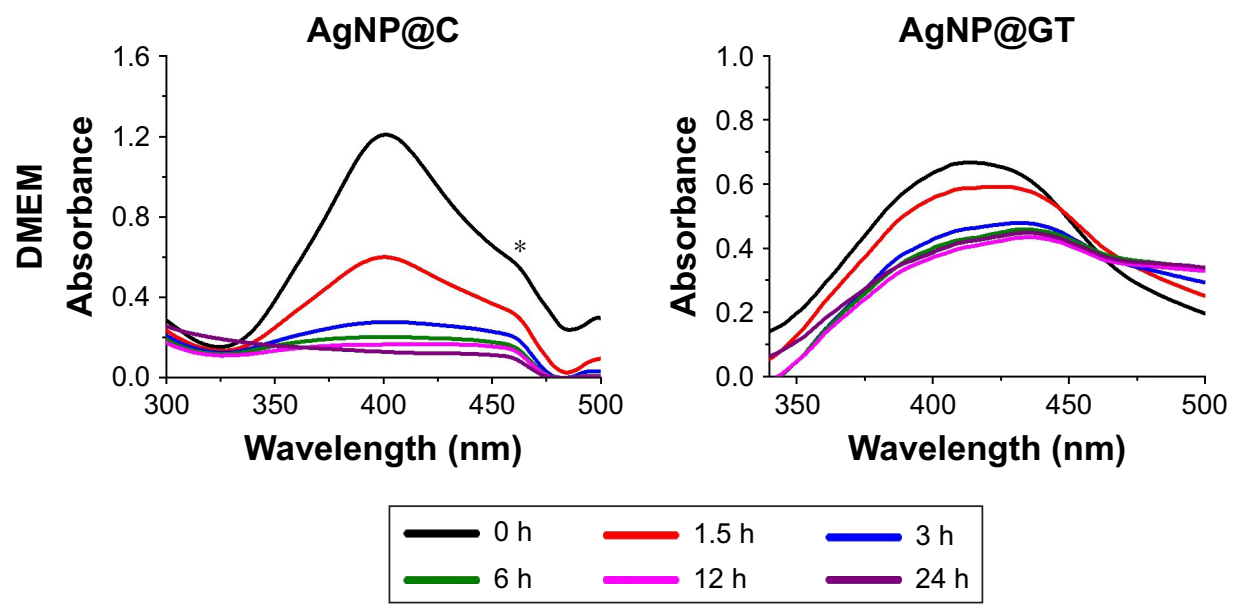

Figure S5 UV-Vis spectral changes of the as-prepared silver nanoparticles (citrate-stabilized AgNP@C, green tea-stabilized AgNP@GT) in the presence of DMEM.*marks a UV-Vis detection error during the measurements that should be disregarded.

Abbreviations: AgNP@C, citrate-capped nanosilver; AgNP@GT, green tea extract-stabilized silver nanoparticle.

\section{References}

1. Avnir D. Recent progress in the study of molecularly doped metals. Adv Mater. 2018;30(41).

2. Naor H, Avnir D. Electroless functionalization of silver films by its molecular doping. ACS Appl Mater Interfaces. 2015;7(48):26461-26469.
3. Kühn M, Ivleva NP, Klitzke S, Niessner R. Investigation of coatings of natural organic matter on silver nanoparticles under environmentally relevant conditions by surface-enhanced Raman scattering. Sci Total Env. 2015;535:122-130.
International Journal of Nanomedicine

\section{Publish your work in this journal}

The International Journal of Nanomedicine is an international, peerreviewed journal focusing on the application of nanotechnology in diagnostics, therapeutics, and drug delivery systems throughou the biomedical field. This journal is indexed on PubMed Central, MedLine, CAS, SciSearch ${ }^{\circledR}$, Current Contents ${ }^{\circledR} /$ Clinical Medicine,

\section{Dovepress}

Journal Citation Reports/Science Edition, EMBase, Scopus and the Elsevier Bibliographic databases. The manuscript management system is completely online and includes a very quick and fair peer-review system, which is all easy to use. Visit http://www.dovepress.com/ testimonials.php to read real quotes from published authors. 